\title{
Vers un scénario « Des usages agro-écologiques des terres pour une alimentation diversifiée et de qualité et un système alimentaire territorialisé » en Tunisie en 2050
}

\author{
Marie de Lattre-Gasquet ${ }^{1, *}$, Clémence Moreau ${ }^{2}$, Mohamed Elloumi $^{3}$ et Leïth Ben Becher ${ }^{4}$ \\ ${ }^{1}$ Cirad, coordinateur Cirad de l'équipe projet Agrimonde-Terra \\ ${ }^{2}$ Cirad, membre de l'équipe projet Agrimonde-Terra, Cirad \\ ${ }^{3}$ INRAT, membre du Comité des scénarios d'Agrimonde-Terra \\ ${ }^{4}$ Synagri, membre du Comité des scénarios d'Agrimonde-Terra
}

Reçu le 31 janvier 2017 - Accepté le 24 avril 2017

\begin{abstract}
Résumé - À l'initiative de l'Institut national de recherche agronomique de Tunisie (INRAT) et de l'équipe Agrimonde-Terra, et avec le Syndicat des agriculteurs de Tunisie (Synagri), en mars 2015, un atelier de construction de scénarios d'usages des terres a réuni une vingtaine de personnes, acteurs publics, parapublics et représentants de la société civile afin de construire des scénarios d'usages des terres en Tunisie. Quatre scénarios ont été construits dont un scénario tourné vers l'agro-écologie. Nous explicitons ici la méthode suivie et les conséquences du scénario «Des usages agro-écologiques des terres » pour les usages des terres et la sécurité alimentaire. Nous montrons les tendances favorables et hostiles à l'évolution vers cette trajectoire. Nous décrivons le chemin d'impact du processus de prospective.
\end{abstract}

Mots clés : prospective / usage des terres / sécurité alimentaire / Tunisie / chemin d'impact

\begin{abstract}
Towards a scenario "Agro-ecological land use for diversified and quality food and a localized food system" in Tunisia in 2050. At the initiative of the National Agronomic Research Institute of Tunisia (INRAT) and the Agrimonde-Terra team, and with the Tunisian Farmers' Union (Synagri), a workshop on land use and food security in Tunisia in 2050 took place in March 2015. About 20 participants from public, para public organizations and from civil society discussed for two days and constructed four scenarios using the results of the Agrimonde-Terra foresight process. These scenarios include one directed towards agro-ecological practices. We explain here the method used to build the scenarios as well as the consequences of the "Agro-ecological uses of land and food quality", scenario for land use and food security. We show the trends that favor this trajectory and those which do not. We describe the impact trajectory of the foresight process.
\end{abstract}

Keywords: foresight / land use / food security / Tunisia / impact pathway

\section{Pourquoi une réflexion prospective sur les usages des terres et la sécurité alimentaire en Tunisie?}

La Tunisie couvre une superficie d'environ 16,21 millions d'hectares pour une population estimée en 2014 à 11 millions d'habitants avec un taux de croissance naturel de l'ordre de $1 \%$. La population est très jeune et le taux d'urbanisation

\footnotetext{
* Auteur de correspondance :

marie.de_lattre-gasquet@cirad.fr
}

(près de $70 \%$ ) est en constante augmentation. La population se concentre le long du littoral et exerce une pression importante sur les ressources en eau et en terre aux dépens de l'agriculture (Chabbi, 2006). Le produit intérieur brut (PIB) est en progression constante mais sa distribution par habitant est inégale, son niveau par habitant étant inférieur dans les zones rurales. L'économie se caractérise par la prépondérance du secteur tertiaire et l'agriculture contribue à hauteur de 8 à $12 \%$ du PIB selon les années et emploie $17 \%$ de la main d'œuvre. Le secteur agricole demeure néanmoins très important notamment pour l'emploi, les revenus des plus pauvres et les exportations; il joue un rôle de filet de sécurité social dans 
certaines régions du pays. L'agriculture est assurée à travers 516000 exploitations d'une taille moyenne de 10 ha avec environ $75 \%$ des exploitants disposant d'une superficie de moins de 10 ha. L'élevage constitue le tiers du PIB agricole et le secteur irrigué $37 \%$ du PIB agricole (Gafrej, 2016). Par ailleurs, les forêts sont d'une importance capitale pour l'économie tunisienne. Densément peuplées, elles participent néanmoins à $14 \%$ au bilan énergétique national et $17 \%$ des besoins du cheptel national produits par les parcours forestiers (Gafrej, 2016) ; elles fournissent d'importants services environnementaux.

Néanmoins, le développement de l'agriculture tunisienne se heurte aujourd'hui à un certain nombre de contraintes structurelles. En particulier, le modèle agronomique rencontre des contraintes de croissance et de stabilisation de la production tant en sec qu'en irrigué, et l'agriculture risque des pertes pouvant atteindre plus de $20 \%$ du PIB agricole en période de sécheresse. La pression sur les ressources naturelles, en particulier les sols et l'eau, est de plus en plus forte et entraîne une dégradation à la limite de la réversibilité et de la capacité de résilience de certains écosystèmes. Les investissements publics stagnent et il n'y a pas d'investissements privés significatifs. La profession et les filières sont mal organisées, et le cadre institutionnel est peu performant. Les structures agraires, avec la coexistence de deux types de structures d'exploitations, limitent les évolutions (Ministère de l'Agriculture et de l'Environnement de Tunisie et al., 2011). L'agriculture et la société rurale traversent une crise profonde depuis le début des années 2000, et la révolution de janvier 2011 peut être interprétée comme une crise de l'agriculture familiale et des limites de ses modes d'adaptation (Elloumi, 2015). La part des produits agricoles importés dans la consommation alimentaire (en calories) est d'environ $40 \%$ et croit depuis le début des années 1960 (Marty, 2015). La Tunisie est autosuffisante en lait et en fruits et légumes, mais elle importe du blé, de l'orge, du maïs, de l'huile de soja, du sucre raffiné, des tourteaux de soja, et des pommes de terre (Marx et Fouquet, 2013). Grâce aux importations, la disponibilité alimentaire a fortement augmenté entre 1960 et 2010, passant d'environ 2000 kilocalories par habitant et par jour à environ 3300 kilocalories par habitant et par jour. Cette transition alimentaire et nutritionnelle a conduit à une forte croissance de l'obésité (Badran et Laher, 2011) et des carences nutritionnelles. Ces tendances sont-elles irréversibles? D'autres trajectoires sont-elles imaginables? Si oui, quelles sontelles? Comment y parvenir?

La prospective n'a pas pour objet de prédire l'avenir mais de nous aider à le construire. Elle nous invite donc à le considérer comme à faire, à bâtir, plutôt que comme quelque chose qui serait déjà décidé et dont il conviendrait seulement de percer le mystère (de Jouvenel, 2004). La prospective est « une approche systématique, participative et pluridisciplinaire pour explorer les futurs à moyen et long-terme et les facteurs de changement» (FTP, 2014). Elle signifie que «l'avenir est pour l'homme, en tant que sujet connaissant, domaine d'incertitude, et pour l'homme, en tant que sujet agissant, domaine de liberté et de puissance » (de Jouvenel, 1964). Ou, pour citer Gaston Berger, père de la prospective en France, il s'agit de « considérer l'avenir non plus comme une chose déjà décidée et qui, petit à petit, se découvrirait à nous, mais comme une chose à faire » (Berger, 1958). Face à l'avenir, les hommes ont le choix entre quatre attitudes: la passivité (comme l'autruche qui subit le changement), la réactivité (comme le pompier qui attend que le feu soit déclaré pour le combattre), la pré-activité (comme l'assureur qui se prépare aux changements prévisibles) et la proactivité (comme celui qui agit pour provoquer les changements souhaités) (Godet, 1997). Mais comme « les meilleures idées sont celles que l'on découvre soimême» (Godet, 1997), c'est par l'appropriation intellectuelle et affective de la réflexion prospective que l'anticipation peut servir à l'action et la décision.

C'est dans cet esprit qu'ont été conduit, par le Cirad et l'Inra, le processus de prospective sur les usages des terres et la sécurité alimentaire en 2050 appelé Agrimonde-Terra ainsi que l'atelier de construction de scénarios d'usages des terres en Tunisie. Cet atelier a été organisé à l'initiative de l'Institut national de recherche agronomique de Tunisie (INRAT) et d'AgrimondeTerra, et avec le Syndicat des agriculteurs de Tunisie (Synagri). Il a eu lieu en 2015 et constituait pour l'équipe Agrimonde-Terra un test de la facilité d'appropriation des résultats de la réflexion prospective Agrimonde-Terra par des décideurs nationaux et de leur utilisation pour construire des scénarios qui pourraient contribuer à changer les usages des terres et la sécurité alimentaire à l'échelle nationale. Au cours de cet atelier, les participants ont construit quatre scénarios.

Nous présenterons ici les outils mis au point par Agrimonde-Terra, la méthode utilisée pour construire les scénarios en Tunisie, les quatre scénarios qui ont été construits puis comparés. Les participants tunisiens à l'atelier se sont facilement appropriés les résultats d'Agrimonde-Terra. L'un des scénarios qu'ils ont construit - appelé «Des usages agroécologiques des terres pour une alimentation diversifiée et de qualité et un système alimentaire territorialisé » - leur est apparu comme une trajectoire favorable pour le système agroalimentaire tunisien, les usages des terres et la sécurité alimentaire. Les tendances actuelles vers cette trajectoire ainsi que celles qui gênent son développement sont identifiées ${ }^{1}$. Ce test de l'appropriation des scénarios Agrimonde-Terra à l'échelle nationale a également permis de progresser dans l'analyse du chemin d'impact de la prospective.

\section{Agrimonde-Terra}

Agrimonde-Terra est un exercice de prospective sur les usages des terres et la sécurité alimentaire en 2050 mené à l'initiative du Cirad et de l'Inra, de façon collective, par une équipe projet, des chercheurs et un comité des scénarios. Les principaux résultats sont présentés dans un document intitulé "Agrimonde-Terra: foresight land use and food security in 2050. Short report of the foresight» (Cirad et Inra, 2016). Au cours du processus de prospective ont été construits :

- un cadre conceptuel qui représente le système « usages des terres et sécurité alimentaire ». Ce système est complexe et

\footnotetext{
${ }^{1}$ Cet article est une version améliorée et approfondie d'un document de travail : de Lattre-Gasquet et Moreau (2016). «Un test de l'outil de dialogue Agrimonde-Terra : la construction de scénarios d'usages des terres en 2050 avec des décideurs tunisiens. » Document de travail. Prospective Agrimonde-Terra : usage des terres et sécurité alimentaire en 2050. Juin 2016.
} 


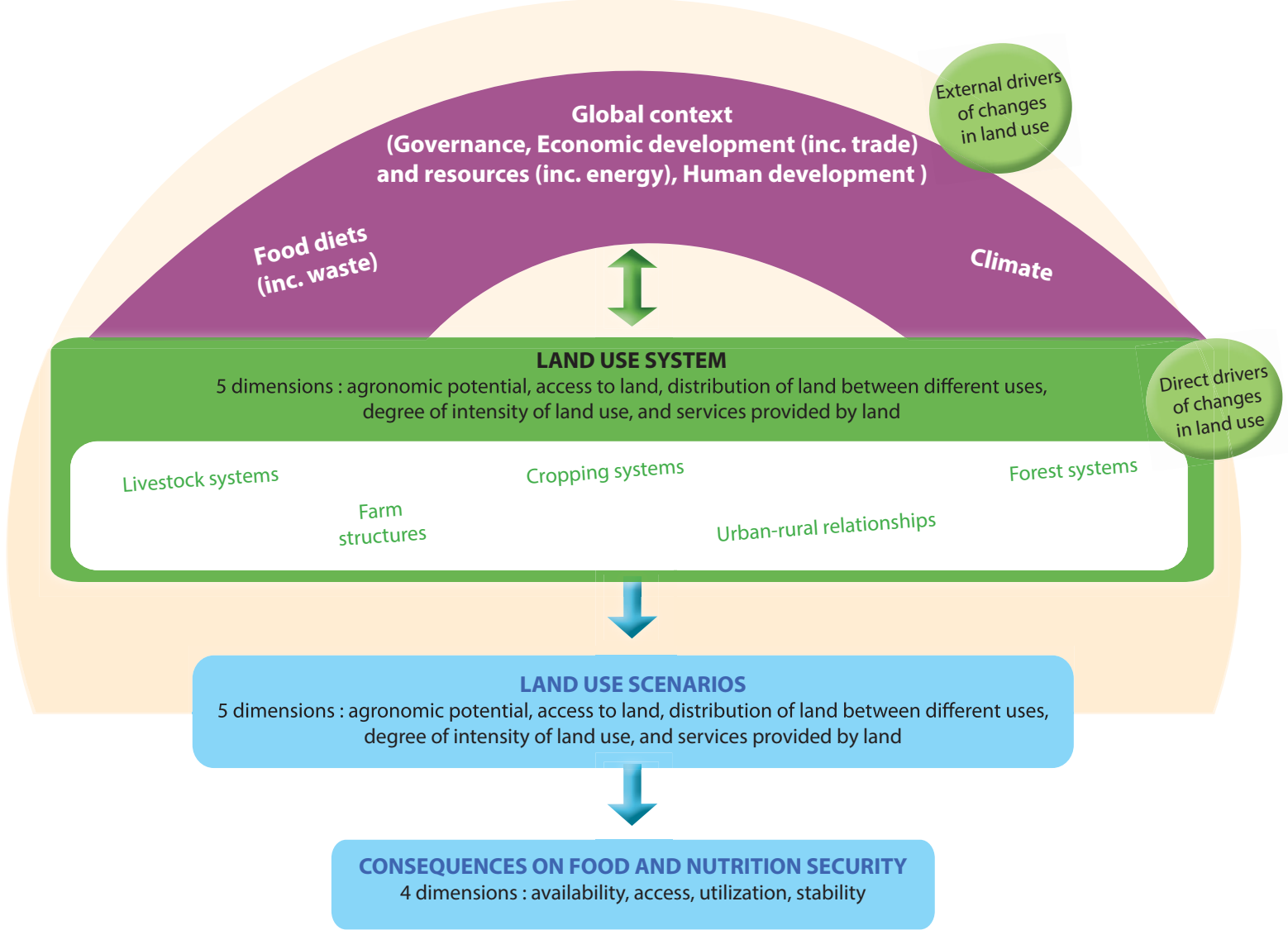

Fig. 1. Le système « Usage des terres et sécurité alimentaire » d'Agrimonde-Terra.

dynamique. Dans la Figure 1, les changements d'usages des terres résultent d'interactions complexes entre des causes directes et externes, et ont des conséquences sur la sécurité alimentaire, qui vont de l'échelle locale à l'échelle mondiale. Il existe donc des liens entre les différentes causes de changements, certaines causes peuvent avoir des effets à court terme sur les usages des terres alors que d'autres ont des effets à très long terme, et des boucles de rétroaction régulent le système. Les activités agricoles et forestières, c'est-à-dire les systèmes de cultures, les systèmes d'élevage et les systèmes forestiers font partie des causes directes de changement des usages des terres, ainsi que les structures de production et les relations entre les zones urbaines et rurales. Le changement climatique, les régimes alimentaires et le contexte mondial, régional et national - à savoir la géopolitique et la gouvernance, le développement économique (y compris le commerce) et les ressources (y compris l'énergie), ainsi que le développement humain - sont les causes externes de changement des usages des terres. Elles interagissent entre elles ainsi qu'avec les causes directes de changement. Par exemple, les régimes alimentaires ont une influence sur les systèmes de cultures et d'élevage, mais sont aussi influencés par eux;

- un tableau morphologique qui représente les hypothèses qualitatives d'évolution possible des causes externes et directes de changements des usages des terres (Tab. 1);
- cinq scénarios contrastés d'usages des terres et de sécurité alimentaire qui ont été construits de manière cohérente, rigoureuse et transparente en combinant les hypothèses relatives à chaque cause directe et indirecte de changement. Ils décrivent le cheminement entre la situation actuelle et 2050 en suivant l'évolution des principales causes directes et externes des changements des usages des terres. Ils présentent également les conséquences des évolutions sur les cinq dimensions des usages des terres et les quatre dimensions de la sécurité alimentaire et nutritionnelle. Ces cinq scénarios sont appelés: «Des usages des terres pilotés par la métropolisation» «Des usages des terres pour des systèmes alimentaires régionaux » «Des usages des terres orientés par des ménages pluriactifs et mobiles» «Des usages des terres pour une alimentation de qualité et une nutrition saine» et «Des terres en tant que communs des communautés rurales dans un monde fragmenté»;

- un modèle de bilans adapté pour la prospective Agrimonde-Terra, appelé GlobAgri-AgT. GlobAgri-AgT a été utilisé pour simuler les impacts des scénarios sur les surfaces, la production et les échanges de 14 régions, dont l'Afrique du Nord.

Ce sont les résultats de ce processus de réflexion prospective - à son stade d'avancement de début 2015 mené par le collectif Agrimonde-Terra qui ont servi de base au travail de construction des scénarios en Tunisie. 
Tableau 1. Agrimonde-Terra: hypothèses pour 2050 pour chaque cause d'évolution du système usage des terres et sécurité alimentaire

\begin{tabular}{|c|c|c|c|c|c|c|c|c|c|c|}
\hline \multirow[b]{2}{*}{ Global Context } & \multicolumn{10}{|c|}{ Alternative assumptions for 2050} \\
\hline & \multicolumn{2}{|l|}{$\begin{array}{l}\text { Sustainable and } \\
\text { cooperative world }\end{array}$} & \multicolumn{2}{|c|}{$\begin{array}{l}\text { Regionalization and } \\
\text { energy transition }\end{array}$} & \multicolumn{2}{|c|}{$\begin{array}{l}\text { Economic and } \\
\text { political } \\
\text { fragmentation }\end{array}$} & \multicolumn{3}{|c|}{$\begin{array}{l}\text { Conventional } \\
\text { development by } \\
\text { market forces }\end{array}$} & Non-State actors \\
\hline $\begin{array}{l}\text { Climate } \\
\text { Change }\end{array}$ & \multicolumn{3}{|c|}{ Stabilization of global warming } & \multicolumn{3}{|c|}{ Moderate warming } & \multicolumn{4}{|c|}{ Runaway climate change } \\
\hline Food Diets & \multicolumn{2}{|c|}{$\begin{array}{c}\text { Transition to diets based } \\
\text { on ultra-processed } \\
\text { products }\end{array}$} & \multicolumn{3}{|c|}{$\begin{array}{l}\text { Transition to diets based } \\
\text { on animal products }\end{array}$} & \multicolumn{3}{|c|}{$\begin{array}{l}\text { Healthy diets based on food } \\
\text { diversity }\end{array}$} & \multicolumn{2}{|c|}{$\begin{array}{l}\text { Regional diversity of diets } \\
\text { and food systems }\end{array}$} \\
\hline $\begin{array}{c}\text { Urban } \\
\text { - Rural } \\
\text { Relationships }\end{array}$ & \multicolumn{2}{|c|}{$\begin{array}{l}\text { Large metropolitan } \\
\text { region }\end{array}$} & \multicolumn{3}{|c|}{$\begin{array}{l}\text { Multilocal and } \\
\text { multi-active households in } \\
\text { rural-urban archipelagos }\end{array}$} & \multicolumn{3}{|c|}{$\begin{array}{c}\text { Rural areas integrated within } \\
\text { urban networks through } \\
\text { value chains }\end{array}$} & \multicolumn{2}{|c|}{$\begin{array}{c}\text { Urban fragmentation and } \\
\text { counter-urbanization }\end{array}$} \\
\hline $\begin{array}{l}\text { Farm } \\
\text { Structures }\end{array}$ & $\begin{array}{l}\text { Marginalized farms } \\
\text { for a livelihood } \\
\quad \text { survival }\end{array}$ & $\begin{array}{r}\mathrm{H} \\
\mathrm{st} \\
\text { agrc }\end{array}$ & $\begin{array}{l}\text {-run } \\
\text { y for } \\
\text { stment }\end{array}$ & & $\begin{array}{l}\text { lent farms } \\
\text { amercial } \\
\text { dency }\end{array}$ & $\begin{array}{r}\text { Farms } \\
\text { goods a } \\
\text { to sur } \\
\text { com }\end{array}$ & $\begin{array}{l}\text { ucing } \\
\text { ervices } \\
\text { ding } \\
\text { ity }\end{array}$ & $\begin{array}{l}\mathrm{Ag} \\
\mathrm{coc} \\
\mathrm{em}\end{array}$ & $\begin{array}{l}\text { ultur } \\
\text { rativ } \\
\text { asizir } \\
\text { ality }\end{array}$ & $\begin{array}{l}\text { Resilient farms } \\
\text { embedded in } \\
\text { urban processes }\end{array}$ \\
\hline Livestock Systems & $\begin{array}{l}\text { Backyard } \\
\text { livestock }\end{array}$ & & $\begin{array}{l}\text { onventi } \\
\text { nsive liv } \\
\text { local re }\end{array}$ & $\begin{array}{l}\text { al } \\
\text { tock } \\
\text { urces }\end{array}$ & $\begin{array}{l}\text { Conven } \\
\text { intensive } \\
\text { with im } \\
\text { resou }\end{array}$ & $\begin{array}{l}\text { tional } \\
\text { livestock } \\
\text { ported } \\
\text { rces }\end{array}$ & $\begin{array}{r}\text { Agro- } \\
\text {-stock } \\
\text { nergy } \mathrm{u} \\
\text { or u }\end{array}$ & $\begin{array}{l}\text { ogice } \\
\text { land } \\
\text { agric } \\
\text { nizat }\end{array}$ & $\begin{array}{l}\text { ive } \\
\text { sy- } \\
\text { ture }\end{array}$ & $\begin{array}{l}\text { Livestock on } \\
\text { marginal land }\end{array}$ \\
\hline Cropping Systems & $\begin{array}{c}\text { Collapse of cropr } \\
\text { systems }\end{array}$ & & & $\begin{array}{l}\text { onvent } \\
\text { ensific }\end{array}$ & & & $\begin{array}{l}\text { ainable } \\
\text { ification }\end{array}$ & & & gro-ecology \\
\hline
\end{tabular}

\section{La construction des scénarios d'usages des terres en Tunisie à l'aide d'Agrimonde- Terra}

À l'initiative de l'INRAT ${ }^{2}$ et d'Agrimonde-Terra et avec le Synagri $^{3}$, un atelier de construction de scénarios d'usages des terres et d'étude de leurs conséquences sur la sécurité alimentaire a eu lieu à Tunis les 11 et 12 mars 2015. Il a rassemblé une vingtaine de participants tunisiens (représentants d'agriculteurs, de Ministères, de l'Industrie agroalimentaire et chercheurs) et des représentants de trois agences

\footnotetext{
${ }^{2}$ En particulier, Mohamed Elloumi (INRAT), membre du comité des scénarios d'Agrimonde-Terra, qui préside un Think Tank dédié à la réflexion sur les politiques agricoles en Tunisie.

${ }^{3}$ En particulier, Leïth Ben Becher, Président du SYNAGRI, membre du comité des scénarios d'Agrimonde-Terra.
}

d'aide au développement choisis par les organisateurs tunisiens ${ }^{4}$. L'initiative était technique - il s'agissait d'une part, de tester l'appropriation des scénarios Agrimonde-Terra à l'échelle nationale et d'autre part, de mieux identifier leur utilité pour la décision publique et privée et les participants n'étaient pas des responsables politiques.

\footnotetext{
${ }^{4}$ Les participants étaient : Naziha Atti, INRAT ; Haithem Bahri, INRGREF ; Leïth Ben Becher, SYNAGRI ; Rabaa Ben Salah, DGPA ; Zied Ben Youssef, GDA Sicilo-Sarde; Aziz Bouhejba, APAD et agriculteur ; Karim Daoud, GDA GERT ; Leïla Douggui Bensalem, Technopôle de Bizerte ; Mohamed Elloumi, INRAT ; Marie-Aude Even, FAO ; Abdelkader Hamdane, INAT ; Mohamed Larbi Bourguira, ATIA ; Raouf Laajimi, ONAGRI ; Rabah Lahmar, CIRAD ; Mathieu Le Grix, AFD ; Noureddine Nasr, FAO ; Samira Nefzi, OTEDD; Denis Pommier, Commission Européenne ; Anis Sendi, Association des jeunes agriculteurs de Gafsa. Le directeur général de l'INRAT, M. Mohamed Ben Hamouda, a ouvert l'atelier.
} 
La construction des scénarios d'usages des terres s'est déroulée en cinq étapes :

- un diagnostic partagé des usages des terres passés et actuels en Tunisie;

- une appropriation des hypothèses relatives au contexte mondial et au changement climatique de la réflexion prospective Agrimonde-Terra, la construction d'hypothèses relatives à la gouvernance en Tunisie;

- une adaptation des hypothèses de la prospective Agrimonde-Terra relatives aux régimes alimentaires et aux causes directes des changements d'usages des terres à la situation de la Tunisie;

- la construction de scénarios d'usages des terres et sécurité alimentaire pour la Tunisie;

- une analyse comparative des quatre scénarios.

\subsection{Première étape: un diagnostic partagé des usages des terres passés et actuels en Tunisie}

L'équipe Agrimonde-Terra responsable de l'atelier Tunisie avait fait une analyse rétrospective ${ }^{5}$, sur les 30 dernières années, du système «Usage des terres et sécurité alimentaire » en Tunisie ainsi qu'un descriptif de la situation en 2015 des cinq dimensions des usages des terres et des quatre dimensions de la sécurité alimentaire (disponibilité, accès, utilisation, stabilité). Cette analyse rétrospective a été présentée à l'ensemble des participants, critiquée et, en moins d'une demi-journée, a permis de parvenir à un diagnostic partagé des cinq dimensions des usages des terres en Tunisie qui sont le potentiel agronomique, l'accès à la terre, le degré d'intensité des usages des terres, la répartition des terres entre différents usages et les services rendus par les terres (de Lattre-Gasquet et al., 2016). Les conclusions de ce diagnostic sont les suivantes :

\subsubsection{Le potentiel agronomique}

Il permet de déterminer l'aptitude des terres à la culture. L'agriculture est pratiquée sur une superficie agricole totale d'environ 10 millions d'ha, soit $62 \%$ de la surface totale. Ces superficies sont réparties entre 5 millions d'hectares de terres labourables, 4 millions d'hectares de parcours naturels et 1 million d'hectares de forêts et de garrigues (ONAGRI, 2016). Cependant, la richesse chimique des sols, leur structure et leur biologie sont en cours de dégradation sous le double effet des variations climatiques (sécheresse, érosion éolienne), des pratiques agricoles et des parcours, diminuant leur capacité de rétention d'eau, ne permettant pas la régénération naturelle et contribuant à l'avancée de la désertification. Le gouvernement tunisien estime que la désertification et la dégradation des sols affectent environ $75 \%$ du territoire national. Chaque année environ 23000 ha sont dégradés, dont 13000 ha de manière quasiment irréversible, soit $0,4 \%$ des terres cultivables. La salinisation des sols et des ressources en eau est également un risque majeur (Zdruli, 2014). Le littoral risque de perdre plus de 100000 ha de terres à cause de sa vulnérabilité à la

\footnotetext{
${ }^{5}$ Moreau et al. (2015). Usages des terres et sécurité alimentaire en 2050. Cas d'étude tunisien. Document de travail. Février 2015.
}

submersion (Gafrej, 2016). Des ressources d'eau souterraine sont disponibles dans le centre et le Sud du pays, mais, si celles du centre sont renouvelables, pour celles du sud il s'agit de ressources non renouvelables et difficilement mobilisables. Dans l'avenir, l'évolution du potentiel agronomique va dépendre des caractéristiques physiques mais également des innovations et des conditions économiques qui permettront soit de restaurer le milieu naturel, soit de s'adapter à sa dégradation.

\subsubsection{L'accès à la terre et les relations de pouvoir qui déterminent l'utilisation des terres}

Malgré plus d'un siècle de réformes foncières et une loi récente sur l'immatriculation des terres et les titres fonciers, l'accès à la terre reste marqué par l'inégalité et l'insécurité (Jouve et Napoléone, 2011). Le dualisme agraire résulte de deux dynamiques: le morcellement des terres et la concentration foncière qui induisent l'augmentation des prix du foncier (Picouet, 2006). L'indivision, l'absence de généralisation du cadastre et la diffusion du faire-valoir indirect avec des baux de très courtes durées alimentent l'insécurité foncière (Amichi et al., 2016). Les terres du domaine privé de l'État constituent un patrimoine important, mais ni une véritable évaluation de leurs usages, ni une planification à moyen et long termes de leur affectation, n'a été faite (Elloumi, 2013). Il existe une discrimination dans l'accès à la terre selon le genre et l'âge qui défavorise les femmes et les jeunes. Les femmes, qui représentent la majeure partie de la main-d'œuvre agricole saisonnière et qui contribuent non seulement à la création de richesses mais aussi à la réduction de la pauvreté dans les régions rurales, souffrent de l'absence de protection sociale et de couverture contre les accidents du travail (Gasmi, 2003 ; Ben Saad, 2014).

\subsubsection{Le degré d'intensité des usages des terres}

La modernisation de l'agriculture, son intensification, est au centre des discours officiels depuis plusieurs décennies. Il s'agissait de transformer les structures de production, les mentalités, pour que les technologies les plus performantes soient appliquées avec efficience et rentabilité. Par conséquent, l'intensification conventionnelle s'est largement répandue, même si une diversité des situations demeure. Près de $8 \%$ de la surface agricole utile (SAU) est irriguée et le modèle agricole est dominé par l'intensification de l'agriculture pluviale (92\% de la SAU). D'après les données de la FAO, l'usage des intrants a été multiplié par quatre depuis 1960. La mécanisation et l'irrigation se sont moins développées, mais le coût environnemental de l'irrigation est de plus en plus élevé du fait des diverses dégradations et surexploitations des ressources observées. La production animale (volailles, aquaculture et bovins) est fortement dépendante des races importées à l'exception des ovins. La production laitière et avicole s'est fortement intensifiée avec abandon des races locales, importation de races étrangères et changements de modes de gestion; cela pose des problèmes de reproduction et conduit à perpétuer les importations de semences et de génisses. L'utilisation de concentrés est de plus en plus fréquente en raison du surpâturage, des sécheresses fréquentes, de l'expansion des cultures de 
céréales et des plantations d'arbres. La culture biologique se développe. Cependant, l'agriculture conventionnelle et les systèmes d'exploitation actuels ont montré leurs limites dans les pays du Maghreb et elles sont mises en cause dans les phénomènes d'érosions hydrique et éolienne, la destruction de la matière organique et de la structure des sols (Zaghouane et al., 2006).

\subsubsection{La répartition des terres entre différents usages}

Les superficies des terres cultivées et des forêts sont relativement stables depuis 1960, mais les terres agricoles subissent de nombreuses dégradations suite à l'envahissement de l'urbanisation, à l'érosion et à l'utilisation de mauvaises techniques du sol et une mauvaise organisation (Roose, 2015). Les cultures s'organisent selon un gradient Nord-Sud qui reflète la succession des zones climatiques: cultures irriguées ou pluviales et forêts dans le Nord du pays, pastoralisme et cultures pluviales ou irriguées, notamment l'arboriculture (olivier) et le maraîchage dans les plaines centrales, et système oasien et des parcours dans le Sud (Fig. 2). Des différences existent également au sein de ces régions. Les cultures pluviales représentent plus de $90 \%$ des surfaces cultivées mais leurs rendements sont faibles. Les surfaces irriguées augmentent lentement, et les superficies laissées en jachère diminuent. Les céréales occupent environ 1,2 million d'hectares (moyenne 2000-2012), soit plus du quart des terres arables et des cultures permanentes; leur production est à $90 \%$ pluviale (Rastoin et Benabderrazik, 2014). La production de graines oléagineuses en Tunisie est assez récente puisque la première expérience remonte aux années 1990 et n'a bénéficié que d'un très faible soutien public. Les surfaces cultivées en arbres fruitiers ont plus que doublé. L'écart important entre le niveau de la consommation et celui de la production nationale conduit la Tunisie à importer de grosses quantités de céréales. Pour la période 2002-2012, en moyenne, le taux de dépendance vis-à-vis des céréales importées a été de près $70 \%$ avec d'importantes fluctuations en fonction de la pluviométrie locale (Rastoin et Benabderrazik, 2014). Avec une proportion importante de ses besoins céréaliers importés, la Tunisie est aujourd'hui importatrice de «terres arables virtuelles ${ }^{6}$.

\subsubsection{Les services rendus par les terres}

L'attention portée aux services rendus par l'agriculture et la forêt commence à se développer (Hassen et Croitoru, 2015 ; Mokhtar, 2013). Dans certains territoires ruraux, les fonctions touristiques et paysagères commencent à être valorisées, et une attention portée à l'installation de jeunes agriculteurs. Mais les emplois agricoles attirent peu, et la main d'œuvre agricole peut manquer à certaines périodes de l'année. L'agriculture tend à dégrader les sols au lieu de lutter contre l'érosion. Dans les régions frontalières, la contrebande se développe en partie pour faire face à la dégradation des revenus et au chômage des ruraux.

\footnotetext{
${ }^{6}$ S'inspirant des travaux sur l'eau effectués en 1993 par Allan (2003) et Van Witzke et Noleppa (2010) ont mené une étude pour mesurer combien de terres « virtuelles » l'Union européenne emploie dans les pays tiers. Nous avons repris ici l'expression « terres virtuelles ».
}

\subsection{Deuxième étape : l'appropriation des hypothèses d'Agrimonde-Terra relatives au contexte mondial et au changement climatique}

Quatre futurs possibles du contexte mondial ainsi que deux hypothèses relatives au changement climatique étaient proposés par Agrimonde-Terra ${ }^{7}$. Les participants à l'atelier ont développé des hypothèses sur l'évolution de la gouvernance en Tunisie. Ces hypothèses sont : politiques volontaristes de développement rural, inertie des problèmes structurels, extraversion de l'économie tunisienne, et faiblesse de l'État et pression de groupes d'intérêt particulier. Pour le changement climatique, les participants n'ont choisi de conserver que l'hypothèse pessimiste (changement climatique accéléré avec une augmentation de la température de 2 à $3{ }^{\circ} \mathrm{C}$ et une baisse de la pluviométrie de 10 à $20 \%$ d'ici 2050) (Giannakopoulos et al., 2009).

\subsection{Troisième étape : l'adaptation à la Tunisie des hypothèses d'Agrimonde-Terra relatives aux régimes alimentaires et aux causes directes des changements d'usages des terres}

Au cours de l'atelier, des groupes de travail accompagnés par un membre de l'équipe Agrimonde-Terra ont discuté de l'analyse rétrospective des cinq causes de changements d'usages des terres (régimes alimentaires, relations entre les zones urbaines et rurales, structures agricoles, systèmes de cultures et systèmes d'élevage) et adapté les hypothèses d'Agrimonde-Terra à la situation de la Tunisie. Il s'agissait de: valider ou modifier l'analyse faite par l'équipe Agrimonde-Terra des tendances lourdes de chaque cause de changement, des incertitudes et des signes de changement, de reformuler les hypothèses ligne par ligne afin de les adapter à la situation de la Tunisie, et de reconstruire des hypothèses pour la Tunisie. Un résumé des analyses et des hypothèses pour la Tunisie est présenté ici.

\subsubsection{Régimes alimentaires}

Les hypothèses d'Agrimonde-Terra ont été adaptées à la situation tunisienne. L'hypothèse tendancielle est celle de la transition alimentaire impulsée par des chaînes de valeur modernes avec consommation de produits ultra-transformés et rôle croissant des firmes multinationales agro-industrielles et des grandes ou moyennes surfaces au détriment des entreprises tunisiennes. La consommation de viande augmente, en fonction du pouvoir d'achat des ménages. L'obésité progresse rapidement (Khamassi et al., 2016). Une hypothèse a été construite pour décrire un autre futur possible: le dualisme alimentaire, qui consiste à la fois en un retour à l'alimentation saine chez les classes urbaines favorisées et à un recours à l'alimentation transformée chez les ruraux et les classes les plus pauvres. Les deux autres hypothèses sont : le sursaut sanitaire, et le régime alimentaire territorialisé avec consommation de produits locaux et différences selon les régions de Tunisie.

\footnotetext{
${ }_{7}^{7}$ L'équipe Agrimonde-Terra et le comité des scénarios ont construit après le mois de février 2015 une $5^{\text {ème }}$ hypothèse de contexte mondial appelée " acteurs non étatiques 》 et une $3^{\text {ème }}$ hypothèse de changement climatique appelée « changement climatique modéré ».
} 


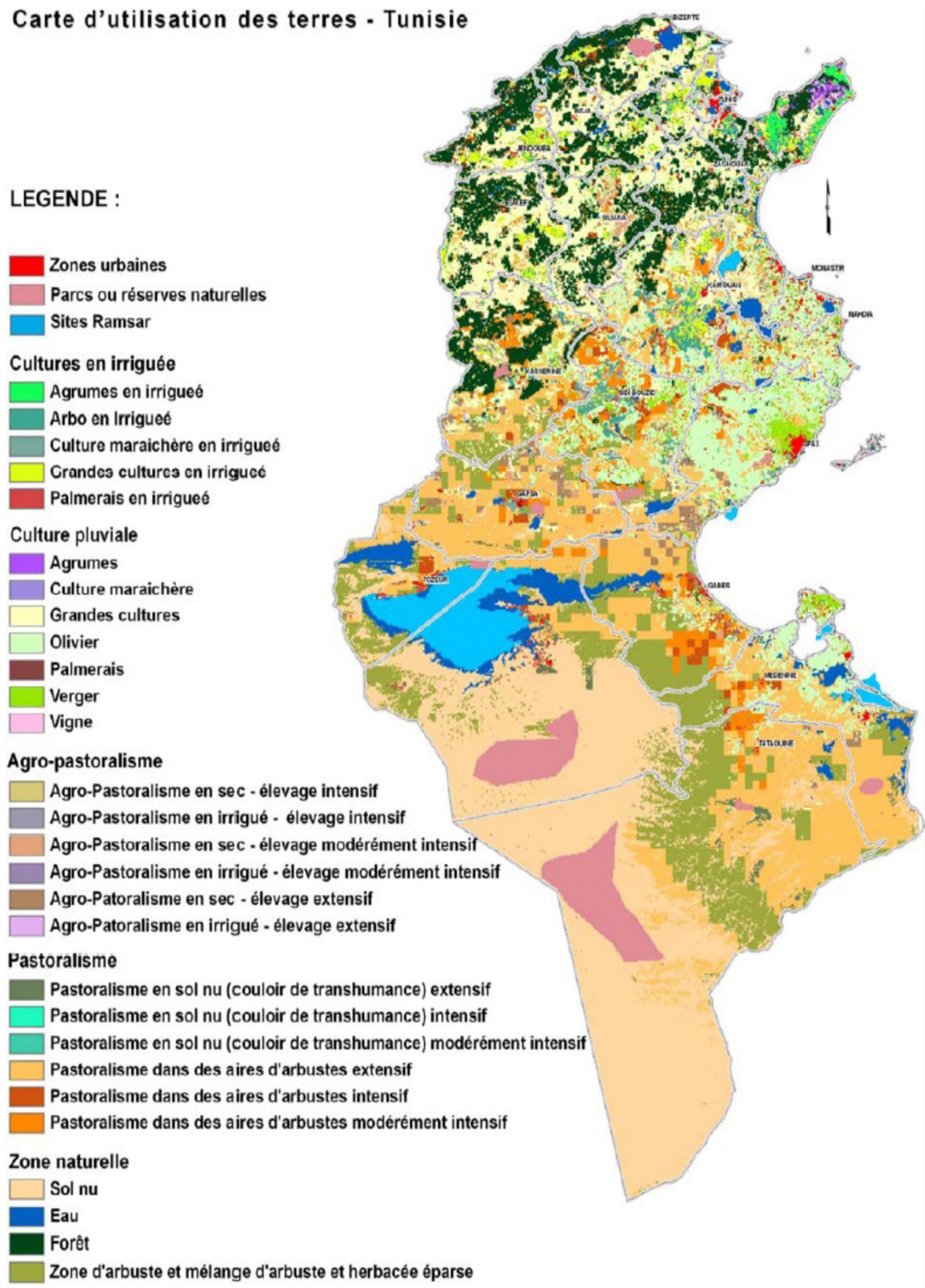

Fig. 2. Carte de l'utilisation des terres en Tunisie. Source : Ministère de l'Agriculture de Tunisie.

\subsubsection{Relations entre les zones urbaines et les zones rurales}

Les hypothèses d'Agrimonde-Terra étaient adaptées à la Tunisie. L'hypothèse tendancielle est celle des ménages multilocalisés et pluriactifs dans un archipel rural-urbain, eu égard à la diffusion de la pluriactivité des ménages ruraux qui s'observe actuellement (Elloumi, 2011). Les hypothèses alternatives sont: les grandes régions métropolitaines et rupture spatiale avec les hinterlands ruraux, les zones rurales intégrées dans des réseaux de villes à travers l'organisation des chaînes de valeur alimentaire, et la fragmentation urbaine et la désurbanisation.

\subsubsection{Les structures agricoles}

Les hypothèses d'Agrimonde-Terra ont été conservées: structures d'exploitation résilientes reliées aux dynamiques urbaines, structures autonomes tout en étant dépendantes des grandes entreprises, structures qui produisent simultanément 
des biens et des services pour la communauté locale, coopératives agricoles tournées vers la qualité, projet agrofinancier à durée déterminée et fermes marginalisées dans une stratégie de survie. L'hypothèse qui décrit le développement d'exploitations capables de résister aux chocs en s'inscrivant dans des dynamiques urbaines a semblé réaliste en Tunisie. Ceci peut être fait soit en renforçant leur insertion dans les marchés urbains grâce à des circuits courts, soit en les encourageant à valoriser leurs produits agricoles, ou à se spécialiser sur des produits de niche, ou à diversifier leurs revenus par d'autres activités non agricoles, ou en fournissant à la fois de la biomasse et des services écosystémiques rémunérés. Il faut noter que quelques mois plus tard, une étude sur la mise à niveau des exploitations agricoles en Tunisie (Doligez, 2015) a fait une typologie des exploitations tunisiennes et identifié quatre types d'exploitations: les moyennes et grandes exploitations pratiquant une agriculture d'entreprise, les moyennes et grandes exploitations à capital familial pratiquant une agriculture patronale à destination des marchés nationaux et internationaux, les petites et moyennes exploitations de type familial et les petites exploitations peu capitalisées à main d'œuvre familiale. Cette typologie ignore les coopératives et met moins l'accent sur l'insertion dans les chaînes de valeur que les hypothèses d'AgrimondeTerra. Cette étude note que les structures capables de produire avec des facteurs de production issus de l'exploitation (fertilisation, fourrage, travail...) permettent d'assurer une robustesse face aux variations des prix que l'on peut attendre (Doligez, 2015).

\subsubsection{Systèmes de cultures}

Agrimonde-Terra a construit quatre hypothèses : intensification conventionnelle, intensification durable, agroécologie, et effondrement des productions végétales. Sous l'hypothèse d'évolution vers «l'intensification durable», les systèmes de cultures continuent à s'intensifier, selon les schémas de l'intensification conventionnelle (simplification et utilisation d'intrants extérieurs à l'exploitation), mais en recherchant une réduction des impacts sur l'environnement. Il y a alors substitutions entre intrants et maximisation de l'efficacité des intrants grâce aux nouvelles technologies. Au contraire, l'évolution des systèmes de cultures vers l'agroécologie implique une refonte complète du système de production et d'exploitation et une recherche d'autonomie $\mathrm{du}$ système. Une hausse du revenu des agriculteurs est recherchée en même temps que la durabilité environnementale. Les participants à l'atelier de Tunis ont choisi de ne pas différencier «intensification durable» et «agro-écologie» et de ne garder que l'hypothèse «agro-écologie» qui comprend des technologies telles que l'agriculture de conservation (Lahmar, 2006). L'approche de l'agro-écologie en Tunisie est pragmatique. Il s'agit avant tout de trouver des alternatives aux pratiques mal raisonnées de l'intensification de l'agriculture, de satisfaire la demande alimentaire et de préserver les ressources naturelles. Elle est promue par des associations telles que l'Association pour une agriculture durable (APAD) et l'Association tunisienne d'agriculture environnementale (ATAE). Les participants à l'atelier ont conservé les hypothèses «effondrement des productions végétales» et « intensification conventionnelle».

\subsubsection{Systèmes d'élevage}

L'élevage joue un rôle clef dans l'économie tunisienne mais la pression du bétail est génératrice de risques importants de dégradation du couvert végétal et des sols et les taux de surpâturage sont entre 70 et $80 \%$ (Halle et al., 2012). Les hypothèses Agrimonde-Terra ont été conservées. L'hypothèse tendancielle est celle de l'élevage intensif conventionnel avec ressources importées qui implique une dépendance aux « terres virtuelles » et une concurrence avec les terres agricoles. Les autres hypothèses sont: l'élevage sur des terres marginales, l'élevage agro-écologique en synergie avec l'agriculture et l'urbanisation, et l'élevage conventionnel avec ressources locales.

Toutes ces hypothèses ont été rassemblées dans un tableau morphologique qui a servi à la construction des scénarios.

\subsection{Quatrième étape : la construction des scénarios d'usages des terres et sécurité alimentaire pour la Tunisie}

La construction de scénarios d'usages des terres et sécurité alimentaire pour la Tunisie s'est faite à partir de la combinaison des hypothèses élaborées pendant l'atelier. Quatre scénarios pour les futurs usages des terres et la sécurité alimentaire en Tunisie ont été construits. Le Tableau 2 représente les hypothèses relatives à chacun d'entre eux. Ces scénarios s'intitulent :

- des usages agro-écologiques des terres pour une alimentation diversifiée et de qualité et un système alimentaire territorialisé (Des usages agro-écologiques des terres);

- dualisme dans l'usage des terres avec cohabitation de deux régimes alimentaires contrastés, de structures et de modes de production (Dualisme dans l'usage des terres);

- spirale de dégradation des terres locales et usages de terres virtuelles (Terres virtuelles);

- des usages des terres pour la survie avec une exacerbation des conflits pour la terre et l'eau (Terres pour survivre et conflits).

\subsubsection{Description du scénario «Des usages agro- écologiques des terres pour une alimentation diversifiée et de qualité et un système alimentaire territorialisé » (voir Tab. 2a).}

\subsubsection{L'élément moteur est le changement des régimes alimentaires}

Ce scénario est impulsé par des changements conséquents dans les régimes alimentaires et par la revalorisation des produits tunisiens. Les consommateurs urbains et ruraux souhaitent éviter les problèmes liés à la suralimentation pour être en meilleure santé ils désirent également avoir davantage accès à des produits agricoles d'origine locale. Ils s'engagent donc dans un consumérisme militant visant à soutenir les agriculteurs tunisiens et à protéger l'environnement. Les institutions en charge de la santé prennent conscience du coût de la prise en charge des maladies liées à la surnutrition et à la malnutrition et orientent leurs actions vers la prévention. Des systèmes alimentaires locaux se mettent en place et permettent de réduire considérablement les importations d'aliments pour 
Tableau 2a. Construction des scénarios « Dualisme dans l'usage des terres » et « Des usages agro-écologiques des terres ».

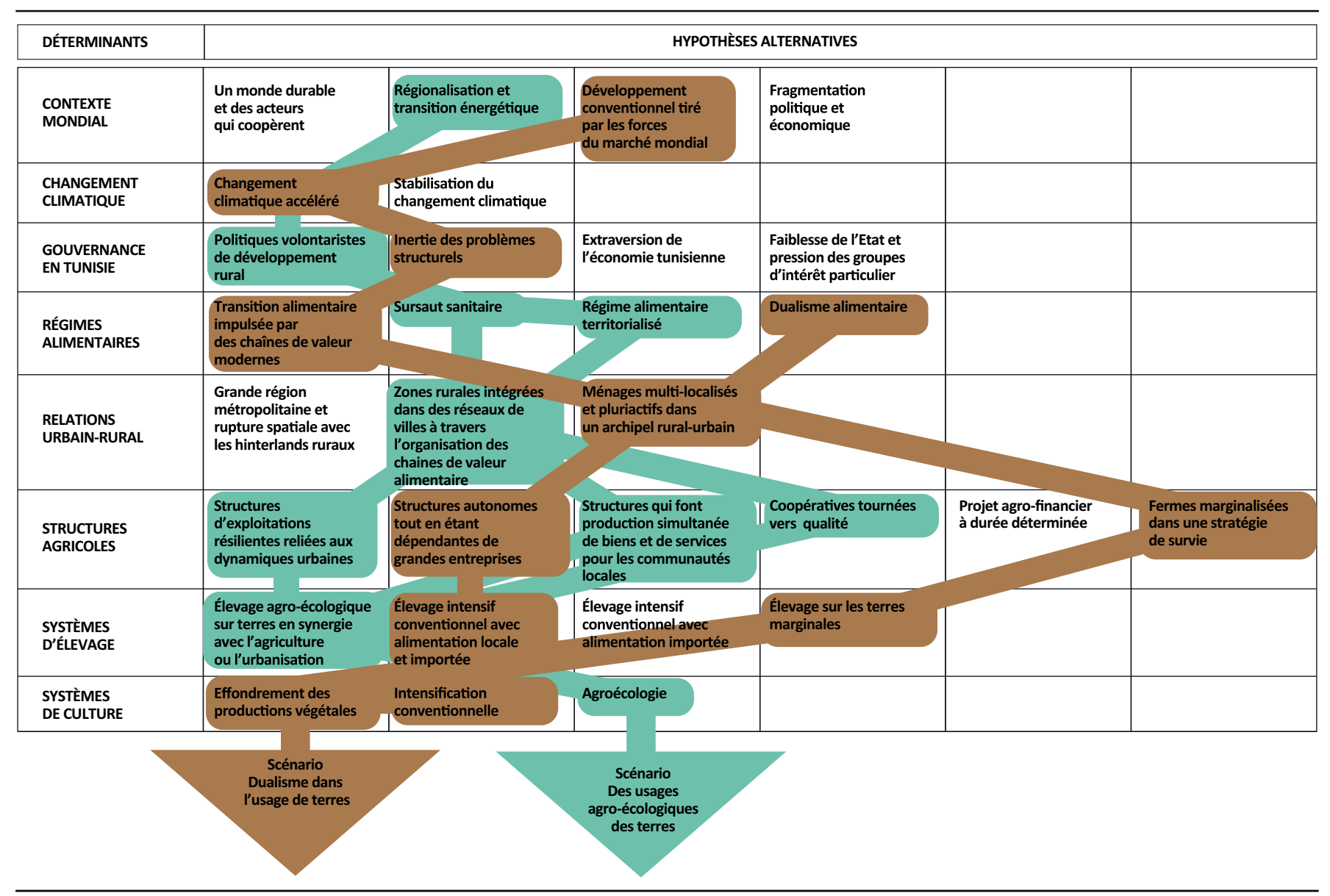

les hommes comme pour le bétail. Les entreprises du secteur agro-alimentaire s'adaptent à ces nouvelles demandes. La labellisation géographique et diététique donne des garanties sur la provenance et l'intérêt nutritionnel des aliments. Par ailleurs, une volonté politique forte permet de sécuriser l'accès à la terre et l'organisation collective des agriculteurs dans le cadre d'une vision stratégique pour le développement rural et agricole.

\subsubsection{Caractéristiques: la transition vers l'agro-écologie}

Les systèmes de cultures et d'élevage évoluent de l'intensification conventionnelle à l'agro-écologie.

Ce scénario implique la valorisation des synergies entre les systèmes d'élevage et de cultures, la recherche d'autonomie quant aux facteurs de production, ainsi que la volonté de limiter l'impact négatif de la production sur l'environnement. Entre 2015 et 2050 , la dynamique la plus déterminante est la transformation des systèmes de cultures et d'élevage. Les exploitations reconçoivent leurs modes de production et les diversifient; elles associent l'agriculture et l'élevage, ce qui permet l'emploi des sous-produits agricoles et des fumures comme fertilisants et aliments pour le bétail. Ces exploitations sont plus diversifiées; elles pratiquent les cultures intercalaires (blé dur - légumineuses dans le Nord ou oliviers, céréales ou fourrages dans le Centre du pays) ainsi que par la diversification des cultures (introduction de nouvelles cultures et exploitation de variétés locales oubliées de colza, tournesol, cactus, féveroles, pois, légumes secs, orge, avoine, vigne, noix, et jatropha). Ces variétés sont mieux adaptées aux sécheresses qui deviennent plus fréquentes en raison du changement climatique. D'autres cultures, comme la betterave à sucre, sont réintroduites afin d'améliorer le taux de couverture en sucre. Le semis direct se développe à l'instar des exploitations qui le pratiquaient déjà en 2015. Les engrais utilisés sont naturels, produits dans la ferme ou achetés à l'extérieur, et donc les exploitations sont plus autonomes. Des solutions sont développées afin de limiter l'impact de l'agriculture sur les ressources, comme l'utilisation d'eaux usées et traitées pour l'irrigation. Les rendements, inférieurs dans un premier temps à ceux de l'agriculture intensive, se stabilisent à un niveau proche de ces derniers, voire supérieur. La qualité des productions s'améliore en termes de nutriments. L'avènement de ce système permet de préserver les ressources naturelles, déjà très lourdement affectées par le réchauffement climatique.

L'objectif poursuivi pour les exploitations est une progression stable des revenus et la réduction des charges pour être plus résilientes aux chocs. Ceci est préféré à l'augmentation des rendements avec une stagnation voire une dégradation des revenus. Pour ce faire, encouragées par l'Association pour la promotion de l'agriculture durable 
(APAD), elles limitent l'utilisation des intrants et commercialisent des produits à haute valeur ajoutée et labellisés (blé dur pour la semoule par exemple). L'État ne peut pas subventionner directement les services environnementaux par manque de moyens mais il incite et encourage la recherche et l'innovation qui renforcent ces évolutions. Les exploitations génèrent également des emplois productifs. Leurs besoins en devises pour importer des produits sont faibles. Les exploitants sont sensibilisés à la durabilité de la gestion des sols et de l'eau, et deviennent des artisans clefs de la sécurité alimentaire nationale. Les liens avec la ville se renforcent, d'une part par la promotion des circuits courts (renforcement du lien entre consommateur et producteur), et d'autre part par la relocalisation des chaînes de valeur alimentaires qui permet aux activités agricoles, de transformation et de vente d'être mieux insérées en milieu rural (production de biens et services). Cette relocalisation des activités de transformation permet le réemploi des rebuts industriels comme aliments de bétail. Certaines exploitations se spécialisent dans les produits de niche pour le marché domestique et l'exportation. D'importants efforts de formation des agriculteurs et des éleveurs sont faits, en particulier pour encourager l'installation de jeunes. Ces formations permettent d'avoir accès à des connaissances et des techniques plus adaptées aux situations géographiques et climatiques particulières. Elles encouragent les interactions entre agriculture et élevage, l'utilisation des effluents et des animaux ainsi que de variétés végétales et de races animales locales améliorées; elles informent en quoi certaines subventions pourraient nuire au développement de produits tels que les légumineuses, le maraîchage et l'arboriculture. Les organisations de producteurs et les services de conseils agricole et rural sont appuyés; la création de coopératives et l'accès au crédit sont facilités. Le passage vers l'agriculture agro-écologique suppose donc une implication renouvelée des acteurs et une coordination de leurs activités, la valorisation et l'exploitation des potentialités tunisiennes, notamment en ce qui concerne les ressources territoriales (paysage, forêt, eau) et la diversité génétique (végétale et animale).

Des recherches et des actions sont menées pour parvenir à des modèles agro-écologiques pour la Tunisie qui permettent de répondre aux enjeux locaux, en particulier l'aridité du milieu et la situation foncière. Par exemple, les espèces locales sont répertoriées et valorisées via une banque de gênes, établie en partenariat avec les pays voisins. Elle permet de développer des plantes résistantes à la sécheresse et à la salinité. L'objectif est de «rusticiser la performance». Des partenariats avec les autres pays voisins sont montés pour créer une banque de gènes et d'échange de plantes. Des initiatives locales se mettent en place: production, échanges et ventes de semences locales, échanges d'expériences entre exploitants. Ces initiatives sont encouragées par l'État et les résultats sont largement diffusés grâce à l'action des vulgarisateurs. Par ailleurs, les subventions sont réorientées vers la production et les exploitations qui développent des cultures à même de restaurer le potentiel agronomique des sols (par exemple la rotation des céréales et des légumineuses, la restauration des terres de parcours), ou qui utilisent les eaux usées, ou qui associent agriculture et élevage, ou qui fournissent des emplois aux jeunes. Des réformes foncières ont également lieu pour que tous aient un accès sécurisé à la terre.

\subsubsection{Conséquences sur la sécurité alimentaire: augmentation de la disponibilité et de la qualité des aliments}

Dans ce scénario, la Tunisie améliore la qualité des aliments produits localement et sa dépendance commerciale augmente peu (voir Sect. 3.5.2). On assiste à un retour à des aliments traditionnels et riches sur le plan nutritionnel, comme les fruits et légumes, les céréales secondaires, la viande de camélidés et l'huile d'olive. C'est une revalorisation de la diète méditerranéenne connue pour ses vertus diététiques. Cette réorientation de l'agriculture et de l'alimentation ne vise pas l'autosuffisance alimentaire, mais encourage les réseaux de clusters agro-alimentaires multi-filières et permet de réduire considérablement les importations de produits tels que les aliments semi-industriels pour le bétail et les huiles.

\subsubsection{Description du scénario «Dualisme dans l'usage des terres avec cohabitation de deux régimes alimentaires contrastés, de structures et de modes de production » (voir Tab. 2a).}

\subsubsection{L'élément moteur est l'absence de réforme foncière}

Ce scénario est caractérisé par la permanence voire le renforcement de l'inégalité d'accès aux terres en l'absence de réforme foncière. En effet, à l'échelle du monde, le développement conventionnel tiré par les forces du marché se poursuit, et en Tunisie, il n'y a pas de réformes refondatrices. Les problèmes structurels qui existaient en 2015 se poursuivent. En ce qui concerne foncier, les freins à une répartition équitable des terres demeurent, (insuffisance du cadastre, importance du parc domanial, difficulté d'accès à la terre pour les femmes et les jeunes). Cette inégalité d'accès aux terres se traduit, sur le long terme par le développement des grandes exploitations d'un côté, et la stagnation voire la régression de la production des petites exploitations de l'autre. La dynamique majoritaire de ce scénario est donc la pérennisation de ces deux modèles divergents, et plus largement, le renforcement des inégalités économiques, sociales et territoriales à l'échelle nationale.

\subsubsection{Caractéristiques: deux modes d'usages des terres et de production de plus en plus contrastés}

Dans ce scénario, les effets du changement climatique s'accentuent et contraignent grandement la production agricole: abandon de certaines terres et diminution des rendements de la plupart des produits cultivés. Face à ces contraintes, deux types de stratégies se développent, compte tenu des priorités et des capacités des exploitations :

Entre 2015 et 2050, le morcellement se poursuit, et les exploitations sont de plus en plus petites ce qui freine la mécanisation et implique une grande vulnérabilité économique. Face aux difficultés pour acquérir des terres, un grand nombre d'exploitants a recours à la location, mais la tension sur le marché implique des baux de plus en plus courts (un an, voire moins). Les exploitants sont marginalisés, fragilisés ; ils pratiquent le nomadisme agricole ce qui accélère la dégradation des terres. Les cultures nécessitant peu d'investissement se développent (céréales, légumineuses) ainsi que 
Tableau 2b. Construction des scénarios «Usages des terres pour survivre et conflits » et « Spirale de dégradation et usages de terres virtuelles ».

\begin{tabular}{|c|c|c|c|c|c|c|}
\hline \multirow{2}{*}{$\begin{array}{l}\text { DÉTERMINANTS } \\
\text { CONTEXTE } \\
\text { MONDIAL }\end{array}$} & \multicolumn{6}{|c|}{ HYPOTHÈSES ALTERNATIVES } \\
\hline & $\begin{array}{l}\text { Un monde durable } \\
\text { et des acteurs } \\
\text { qui coopèrent }\end{array}$ & $\begin{array}{l}\text { Régionalisation et } \\
\text { transition énergétique }\end{array}$ & 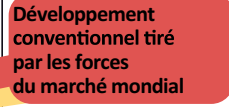 & $\begin{array}{l}\text { Fragmentation } \\
\text { politique et } \\
\text { économique }\end{array}$ & & \\
\hline $\begin{array}{l}\text { CHANGEMENT } \\
\text { CLIMATIQUE }\end{array}$ & $\begin{array}{l}\text { Changement } \\
\text { climatique accéléré }\end{array}$ & $\begin{array}{l}\text { Stabilisation du } \\
\text { changement climatique }\end{array}$ & & & & \\
\hline $\begin{array}{l}\text { GOUVERNANCE } \\
\text { EN TUNISIE }\end{array}$ & $\begin{array}{l}\text { Politiques volontaristes } \\
\text { de développement } \\
\text { rural }\end{array}$ & $\begin{array}{l}\text { Inertie des problèmes } \\
\text { structurels }\end{array}$ & $\begin{array}{l}\text { Extraversion de } \\
\text { I l'économie tunisienne }\end{array}$ & $\begin{array}{l}\text { Faiblesse de l'Etat et } \\
\text { pression des groupes } \\
\text { d'intérêt particulier }\end{array}$ & & \\
\hline $\begin{array}{l}\text { RÉGIMES } \\
\text { ALIMENTAIRES }\end{array}$ & $\begin{array}{l}\text { Transition alimentaire } \\
\text { impulsée par } \\
\text { des chaînes de valeur } \\
\text { modernes }\end{array}$ & Sursaut sanitaire & $\begin{array}{l}\text { Régime alimentaire } \\
\text { territorialisé }\end{array}$ & Dualisme alimentaire & & \\
\hline $\begin{array}{l}\text { RELATIONS } \\
\text { URBAIN-RURAL }\end{array}$ & $\begin{array}{l}\text { Grande région } \\
\text { métropolitaine et } \\
\text { rupture spatiale avec } \\
\text { les hinterlands ruraux }\end{array}$ & $\begin{array}{l}\text { Zones rurales intégrées } \\
\text { dans des réseaux de } \\
\text { villes à travers } \\
\text { l'organisation des } \\
\text { chaines de valeur } \\
\text { alimentaire }\end{array}$ & $\begin{array}{l}\text { Ménages multi-localisés } \\
\text { et pluriactifs dans } \\
\text { un archipel rural-urbain }\end{array}$ & & & \\
\hline $\begin{array}{l}\text { STRUCTURES } \\
\text { AGRICOLES }\end{array}$ & $\begin{array}{l}\text { Structures } \\
\text { d'exploitations } \\
\text { résilientes reliées aux } \\
\text { dynamiques urbaines }\end{array}$ & $\begin{array}{l}\text { Structures autonomes } \\
\text { tout en étant } \\
\text { dépendantes de } \\
\text { grandes entreoprises }\end{array}$ & $\begin{array}{l}\text { Structures qui font } \\
\text { production simultanée } \\
\text { de biens et de services } \\
\text { pour les communautés }\end{array}$ & $\begin{array}{l}\text { Coopératives tournées } \\
\text { vers qualité }\end{array}$ & $\begin{array}{l}\text { Projet agro-financier } \\
\text { à durée déterminée }\end{array}$ & $\begin{array}{l}\text { Fermes marginalisées } \\
\text { dans une stratégie } \\
\text { de survie }\end{array}$ \\
\hline $\begin{array}{l}\text { SYSTÈMES } \\
\text { D'ÉLEVAGE }\end{array}$ & $\begin{array}{l}\text { Élevage agro-écologique } \\
\text { sur terres en synergie } \\
\text { avec l'agriculture } \\
\text { ou l'urbanisation }\end{array}$ & $\begin{array}{l}\text { Élevage intensif } \\
\text { conventionnel avec } \\
\text { alimentation locale } \\
\text { et importée }\end{array}$ & $\begin{array}{l}\text { Élevage intensif } \\
\text { conventionnel avec } \\
\text { alimentation importée }\end{array}$ & $\begin{array}{l}\text { Élevage sur les terres } \\
\text { marginales }\end{array}$ & & \\
\hline $\begin{array}{l}\text { SYSTÈMES } \\
\text { DE CULTURE }\end{array}$ & $\begin{array}{l}\text { Effondrement des } \\
\text { productions végétales }\end{array}$ & $\begin{array}{l}\text { Intensification } \\
\text { conventionnelle }\end{array}$ & Agroécologie & & & \\
\hline & $\begin{array}{l}\text { Scénario } \\
\text { Usages des terres } \\
\text { pour survivre } \\
\text { et conflits }\end{array}$ & & & & & \\
\hline
\end{tabular}

les élevages laitiers avec un très petit nombre d'animaux. Les produits sont destinés au marché local. Leur rentabilité est faible mais ils permettent aux agriculteurs de dégager un revenu quotidien. Dans ces exploitations, l'équilibre économique, agronomique et environnemental est précaire. Pour pallier cette vulnérabilité, les ménages deviennent mobiles et pluriactifs; les ressources gagnées en ville permettent de compenser les revenus agricoles qui sont bas et aléatoires. La ferme est néanmoins un lieu de repli quand la conjoncture urbaine est difficile.

Dans les grandes exploitations, l'intensification conventionnelle continue à être pratiquée. Ces exploitations font l'objet d'investissements importants, que ce soit de la part de structures privées ou de l'État. Elles sont bien intégrées sur le marché international, via l'exportation de leur production, d'une part, et d'autre part, l'achat de semences, d'intrants et de matériel agricole. La production et le cours des produits sont largement dominés par la conjoncture internationale. Les cultures d'exportations (produits maraîchers, dattes, olives) voient leur production augmenter de façon significative. La tendance est à la spécialisation des exploitations et des espèces : le patrimoine génétique de la Tunisie (végétal et animal) est très peu valorisé dans ces exploitations qui sont dans une logique d'intensification et de rente.

\subsubsection{Conséquences sur la sécurité alimentaire: une inégalité croissante}

Les produits transformés et importés forment la base du régime alimentaire. Ils sont achetés dans des grandes surfaces et font l'objet d'incitations publicitaires. En l'absence de réformes, les urbains continuent à bénéficier des subventions à la consommation. Les populations des zones rurales sont de plus en plus marginalisées et souffrent de carences en nutriments et en protéines.

\subsubsection{Description du scénario «Spirale de dégradation des terres locales et usages de terres virtuelles » (voir Tab. 2b).}

\subsubsection{L'élément moteur est l'extraversion de l'économie}

Ce scénario est impulsé par l'extraversion de l'économie agricole tunisienne. Dans un contexte mondial libéral, la Tunisie cherche à s'affirmer dans le marché international dans une optique de division internationale du travail. Elle délaisse 
l'agriculture au profit de l'industrie et des services. De fait, le découplage entre l'acte de produire et de se nourrir s'accentue, ce dont témoigne la balance commerciale toujours plus déficitaire.

\subsubsection{Caractéristiques du scénario: des terres locales en dégradation et un recours accru aux «terres virtuelles »}

Dans ce scénario, afin de satisfaire cette demande alimentaire, la Tunisie a recours à deux types de terres :

- les «terres virtuelles» dont l'importance est croissante. La Tunisie externalise sa production agricole et importe énormément, que ce soit la viande rouge ou les céréales. Ses sources d'approvisionnement dépendent des prix du marché, du contexte géopolitique ou des accords bilatéraux (Tunisie/Union Européenne par exemple). Cependant, étant donné l'accélération du changement climatique à l'échelle mondiale, le marché des produits agricoles est de plus en plus tendu, ce qui rend la Tunisie très vulnérable. Via ces importations d'aliments, ce sont des terres et de l'eau virtuelles qui sont importées;

- les terres situées sur le territoire national qui sont fortement dégradées. Dans une optique de division internationale du travail, la Tunisie se spécialise dans un nombre très restreint de produits : l'huile d'olive, les dattes, l'arboriculture et le maraîchage. L'élevage d'escargots, de lapins ou de criquets se développe ; ils sont vendus à des prix élevés. La tendance est à l'intensification, que ce soit pour les productions végétales ou animales, et seules les exploitations les plus productives persistent. Elles contractualisent avec des entrepreneurs, souvent étrangers. Le niveau global de production diminue, surtout pour l'élevage. L'agriculture tend à devenir une agriculture sans agriculteurs. Les investisseurs agricoles sont principalement des urbains aisés. Une crise rurale importante se produit, avec des migrations vers les métropoles, en particulier Tunis. L'extension urbaine est un facteur important de dégradation des terres. En outre, le changement climatique accéléré entraîne des migrations de populations vers les zones les moins chaudes du pays. Les nappes fossiles du Sud du pays sont exploitées au-delà de leur capacité de renouvellement, ce qui pose des problèmes de pollution et des conflits avec la Libye ou l'Algérie. La spirale de dégradation commence par la terre mais elle va plus loin et concerne l'ensemble des ressources.

\subsubsection{Conséquences sur la sécurité alimentaire: volatilité des prix et insécurité alimentaire des ménages}

En externalisant sa production agricole, la Tunisie externalise également ses risques. Ainsi, elle est de plus en plus vulnérable à la conjoncture économique internationale, et notamment à la volatilité des prix. Cette volatilité n'est guère régulée au niveau international car le contexte est libéral et les instances de régulation sont faibles ou inexistantes. La volatilité est donc compensée par l'État mais son coût est difficilement supportable. La diète tunisienne est de plus en plus basée sur des produits ultra transformés et de la viande, tous deux importés. La Tunisie accueille des filiales des grandes firmes multinationales, bien que celles-ci s'implantent préférentiellement au Maroc. L'agro-industrie à capitaux nationaux en pâtit considérablement. La volatilité des prix nourrit la crainte de voir s'élever une révolte urbaine et populaire (semblable à celle de 2010-2011), ce qui pousse le gouvernement à subventionner largement les produits à la consommation, notamment ceux qui sont néfastes d'un point de vue nutritionnel (huile de graines et sucre). La Tunisie s'expose ainsi à des conséquences sanitaires importantes (d'obésité, de diabète et de cancers).

\subsubsection{Description du scénario «Des usages des terres pour la survie avec une exacerbation des conflits pour la terre et l'eau » (voir Tab. 2b). \\ 3.4.4.1 L'élément moteur est la faiblesse de l'État et les pressions de groupes d'intérêt particulier}

Ce scénario est impulsé par la montée de groupes défendant leurs intérêts particuliers en Tunisie, par exemple des entreprises ou des groupes religieux. Le poids de l'État dans la gouvernance nationale, et en particulier dans le développement agricole et territorial, s'amenuise. Il en résulte une insécurité croissante, qui redessine totalement l'usage des terres en 2050. Cette situation est similaire à celle des autres pays, puisque la tendance globale est à la fragmentation politique et économique.

\subsubsection{Caractéristiques: mitage en zone agricole et dégradation des terres agricoles}

Ce scénario est marqué par trois caractéristiques, qui correspondent à trois étapes de l'évolution de l'usage des terres entre 2015 et 2050 .

Ce scénario est impulsé par une dynamique forte: la précarisation du statut de l'agriculteur, que ce soit au plan économique ou institutionnel. L'agriculture est doublement délaissée, à la fois par le secteur public qui peine à accompagner les petits exploitants, et par les investisseurs privés qui lajuge peu rentable. De fait, seules de petites structures perdurent. La production stagne voire régresse, et étant donné les difficultés d'accès au crédit et à la commercialisation, les agriculteurs se tournent vers les cultures nécessitant peu d'investissement (céréales ou légumineuses en pluvial). La pluriactivité permet de compenser un peu cette fragilité en complétant les revenus, mais l'équilibre des ménages ruraux reste très précaire. Les zones forestières, déjà fragiles en 2015, sont largement concernées par cette précarité. En outre, les difficultés de la reconnaissance du statut de l'agriculteur persistent (pas de statut professionnel, de cadastre et d'impôt foncier) et les rendent vulnérables à l'accaparement des terres et au grignotage des terres par l'urbanisation.

Étant donné l'absence de planification, les terres en milieu rural sont de plus en plus soumises à l'urbanisation, sous forme d'étalement urbain mais aussi de mitage. En outre, les mobilités pendulaires se renforcent, en raison du renchérissement des loyers en ville, de l'amélioration des infrastructures de transport, mais également un désir des tunisiens de vivre «au vert». Il en résulte une interpénétration des zones rurales et urbaines, qui se fait au détriment de l'agriculture.

La précarité de l'agriculture engendre une dégradation rapide des ressources, notamment le sol et l'eau. Cela est empiré par l'accélération du changement climatique. Parallèlement, le mitage urbain implique une concurrence plus dure 
Tableau 3. Évaluation des quatre scénarios pour la Tunisie par les participants à l'atelier.

\begin{tabular}{|c|c|c|c|c|}
\hline $\begin{array}{l}\text { Scénarios } \\
\text { Critères d'évaluation }\end{array}$ & Usages agro écologiques & $\begin{array}{l}\text { Dualisme dans } \\
\text { usages }\end{array}$ & Terres virtuelles & $\begin{array}{l}\text { Terres pour survivre } \\
\text { et conflits }\end{array}$ \\
\hline Rendements des cultures par hectare & ++ & + & +++ & - \\
\hline Utilisation des intrants & + & +++ & - & - \\
\hline Approvisionnement en semences locales & +++ & + & - & + \\
\hline Emplois agricoles & +++ & + & - & + \\
\hline Sécurité alimentaire & $\begin{array}{l}\text { Augmentation de la } \\
\text { disponibilité et de la } \\
\text { qualité des aliments }\end{array}$ & $\begin{array}{l}\text { Inégalités } \\
\text { croissantes }\end{array}$ & $\begin{array}{l}\text { Volatilité des prix. } \\
\text { Insécurité alimentaire } \\
\text { des ménages }\end{array}$ & $\begin{array}{l}\text { Forte insécurité } \\
\text { alimentaire et } \\
\text { nutritionnelle }\end{array}$ \\
\hline
\end{tabular}

sur les ressources, notamment l'eau. L'allocation de l'eau donne la priorité à l'eau potable, mais également à l'eau industrielle ou l'eau dédiée aux activités touristiques, ce qui fait que la situation est largement défavorable à l'agriculture. Ces conflits locaux se doublent d'une insécurité plus générale dans les zones rurales, qui émane de la petite délinquance (vol de bétail par exemple), de menaces terroristes (zones enclavées) ou de risques géopolitiques (frontières avec l'Algérie et la Libye).

\subsubsection{Conséquences sur la sécurité alimentaire: une forte dégradation de la situation alimentaire des ménages}

La petite agriculture, qui pourrait permettre de fournir à l'échelle locale des produits agricoles, et par là assurer la sécurité alimentaire des ménages, n'y parvient pas. L'alimentation tunisienne est largement importée et la diète homogénéisée. En effet, sans soutien politique, la production reste aléatoire et largement dépendante des aléas climatiques. En outre, la précarisation et la marginalisation de l'agriculture en Tunisie rend difficile la commercialisation (absence d'infrastructure de collecte et de distribution, y compris de transports). Ce scénario a un impact négatif sur la sécurité alimentaire.

\subsection{Cinquième étape: Analyse comparative des quatre scénarios}

\subsubsection{L'analyse des participants à l'atelier}

La présentation des quatre scénarios permet de mieux comprendre les conséquences de chacune des trajectoires sur les usages des terres mais également la situation économique, sociale et environnementale et la sécurité alimentaire. Sur la base de son expertise, le groupe a évalué - à dires d'experts les quatre scénarios en indiquant les changements possibles dans les rendements des cultures, l'efficience des productions animales, l'utilisation des intrants, l'approvisionnement en semences locales, l'emploi dans l'agriculture et les activités liées à l'agriculture, la dépendance aux importations, et l'évolution des terres cultivées (Tab. 3).

Les participants ont également travaillé sur les trajectoires des usages des terres et de la sécurité alimentaire dans le temps. Ils ont estimé que le scénario tendanciel de «Dualisme dans l'usage des terres» pourrait se maintenir jusqu'en 2050.
Néanmoins, si le gouvernement tunisien privilégie le commerce international, l'industrie et les services au détriment de l'agriculture, la trajectoire pourrait être modifiée et ce scénario pourrait évoluer vers celui des «Terres virtuelles » où seules les productions agricoles intensives valorisées par le marché mondial et pour lesquelles la Tunisie dispose d'atouts sont privilégiées. Les usages des terres pourraient évoluer vers un scénario encore plus sombre dans lequel la gouvernance est le fait de groupes de pression avec une baisse de l'influence de l'État. On irait alors vers un scénario d'agriculture de subsistance pour les populations rurales dont la vie serait de plus en plus précaire: «Terres pour survivre et conflits». À l'inverse, un sursaut des consommateurs (choix d'un régime alimentaire local et plus sain) et une gouvernance nationale sécurisant encore davantage l'accès à la terre et l'organisation collective, valorisant davantage d'autonomie alimentaire, promouvant les variétés végétales ou animales locales qui pourraient également devenir des niches commerciales sur le marché international, pourraient orienter l'agriculture tunisienne dans une voie de production agricole plus équilibrée entre la production pour l'alimentation locale, la production de spécialités de niche pour l'export et la préservation des ressources locales ; on serait alors dans le scénario «Des usages agro-écologiques des terres ». Ces quatre scénarios ne s'opposent donc pas mais constituent des trajectoires de changement.

Ces quatre scénarios - en particulier le scénario «Des usages agro-écologiques des terres pour une alimentation diversifiée et de qualité et un système alimentaire territorialisé »- permettent de faire ressortir que pour des usages des terres durables et une amélioration de la sécurité alimentaire et nutritionnelle, il faudrait en particulier que :

\subsubsection{Les régimes alimentaires et les chaînes de valeur alimentaires évoluent et que les consommateurs se tournent davantage vers des produits locaux et sains transformés en Tunisie}

Les évolutions des usages des terres seront le résultat de changements dans l'offre mais également dans la demande. Au sein de la demande, le lien entre les régimes alimentaires et les chaînes de valeur est fort. Rastoin et Benabderrazik (2014) soulignent que «la qualité des aliments se construit tout au long des filières. Elle nécessite l'établissement de 
normes de sûreté sanitaire et de qualité nutritionnelle, la mise en place d'un dispositif de contrôle (laboratoire d'analyse), d'incitations et de sanctions, une information claire et complète des consommateurs et un programme de formation à la fois des opérateurs des filières et des consommateurs ». Le système alimentaire deviendra plus autonome et adapté aux ressources naturelles et culturelles régionales si les filières sont ancrées dans les territoires et les zones rurales intégrées dans des réseaux de villes à travers les chaînes de valeur alimentaire.

\subsubsection{L'agriculture soit reconnue comme une profession à part entière}

D'abord pour sécuriser les producteurs et leur permettre d'accéder aux aides et aux soutiens publics et, ensuite, pour éviter que des intrus ou des producteurs «occasionnels» ne détournent ces mêmes aides à leur avantage de façon indue. En outre, l'absence d'un statut définissant le métier d'agriculteur est non seulement perçue comme une injustice supplémentaire faite à la profession (statut fiscal, couverture sociale), mais encore comme une cause de mauvais usage des ressources financières allouées aux agriculteurs (crédits, avantages fiscaux ou financiers, intrants subventionnés, etc.) (Ben Becher, 2016).

\subsubsection{L'agriculture adopte des systèmes de production durable}

Il est urgent de revoir les systèmes de production et d'intégrer dans les stratégies agricoles une approche plus horizontale, axée sur les critères de la durabilité, entendue comme la nécessaire conciliation entre la performance écologique, l'efficacité économique et l'équité sociale. Il s'agit de produire mieux et durablement notamment en introduisant des légumineuses alimentaires et fourragères dans les assolements, en pratiquant les associations de cultures, en ayant une gestion efficiente de l'eau dans le sol et une augmentation de la fertilité des terres par l'agriculture de conservation. Il convient également de renforcer le contrôle phytosanitaire au niveau des frontières pour préserver l'agriculture de l'introduction de nouveaux agents pathogènes et ravageurs dangereux (Ben Becher, 2016).

\subsubsection{La gouvernance des territoires et des structures en charge de l'agriculture soit reconnue comme une question centrale}

Ces questions devraient être discutées avec des acteurs locaux pour approfondir les moyens d'améliorer la sécurité foncière et les voies d'évolution des structures agricoles. Les évolutions des usages des terres doivent être abordées à l'échelle nationale mais également à l'échelle des territoires et des structures, et les liens entre ces échelles doivent être considérés.

À partir de l'analyse des quatre scénarios, les participants à l'atelier ont estimé que le scénario «Des usages agroécologiques des terres pour une alimentation diversifiée et de qualité et un système alimentaire territorialisé » est le plus souhaitable. Il pourrait permettre à la Tunisie d'augmenter la disponibilité alimentaire nationale et la qualité des aliments produits localement pour sa population ainsi que pour les

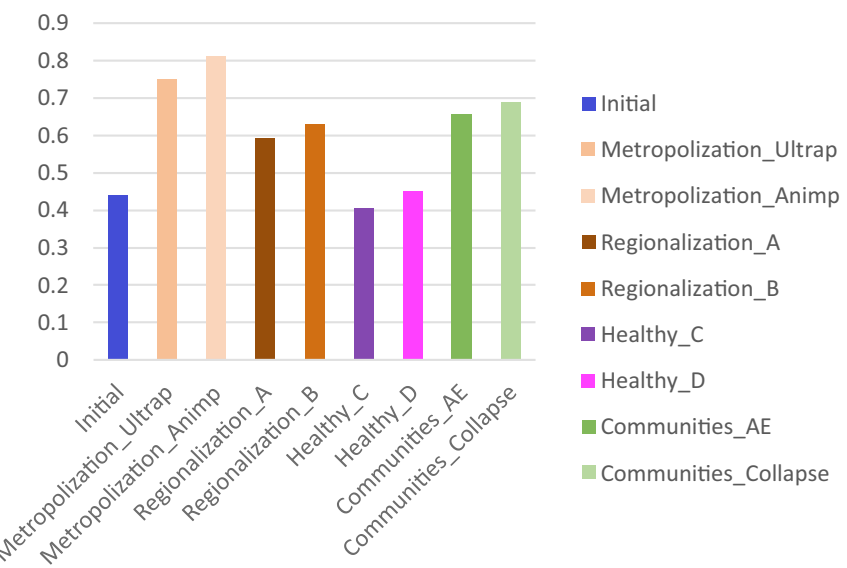

Fig. 3. La dépendance commerciale en Afrique du Nord dans la situation initiale et avec 4 scénarios Agrimonde-Terra. Source : GlobAgri-AgT.

touristes, d'améliorer les chaînes de valeur alimentaires, mais aussi d'assurer des emplois agricoles, de réduire la dégradation des terres et de l'eau, de limiter les coûts de production grâce à la diminution des intrants, et d'assurer aux agriculteurs des revenus complémentaires.

À la fin de la réunion, les participants ont exprimé le souhait d'impliquer des élus et décideurs nationaux dans une future réflexion prospective afin d'induire une transformation dans les usages des terres, et les rendre davantage durables, respectueux des hommes et de leur environnement. Ils ont pensé qu'il serait utile de mener des réflexions prospectives sur les usages des terres avec une variété d'acteurs dans les différentes régions de Tunisie. L'échelle régionale voire locale semble en effet la plus adaptée pour induire un véritable changement dans l'usage des terres.

\subsubsection{Les résultats des simulations des scénarios Agrimonde-Terra en Afrique du Nord}

Un an après l'atelier, les impacts de quatre scénarios Agrimonde-Terra $^{8}$ sur les surfaces, la production et les échanges de 14 régions du monde, dont l'Afrique du Nord, ont été simulés (Le Mouël, 2016). Tous les scénarios ont été simulés avec deux variantes. En particulier, le scénario «Des usages des terres pour une alimentation de qualité et une nutrition saine» (appelé «Healthy» dans les Fig. 3 et 4) a été simulé avec deux variantes ayant trait aux hypothèses d'évolution à 2050 des systèmes de cultures : «intensification durable» (variante dite Healthy_C) et «agroécologie» (dite Healthy_D), les systèmes d'élevage évoluant dans les deux cas vers des systèmes agro-écologiques. Dans Agrimonde-terra, les hypothèses d'évolution des systèmes de cultures sont traduites quantitativement via des hypothèses sur l'évolution des écarts aux rendements potentiels et sur l'évolution de l'intensité culturelle (Le Mouël, 2016). Ainsi, dans la variante Healthy_C (intensification durable), l'écart aux rendements potentiels est réduit de $40 \%$ en

\footnotetext{
${ }^{8}$ Les scénarios sont présentés dans : Cirad and Inra (2016) Agrimonde-Terra: Foresight Land Use and Food Security in 2050. Short Report of the Foresight, June 2016, Paris: Cirad and Inra.
} 


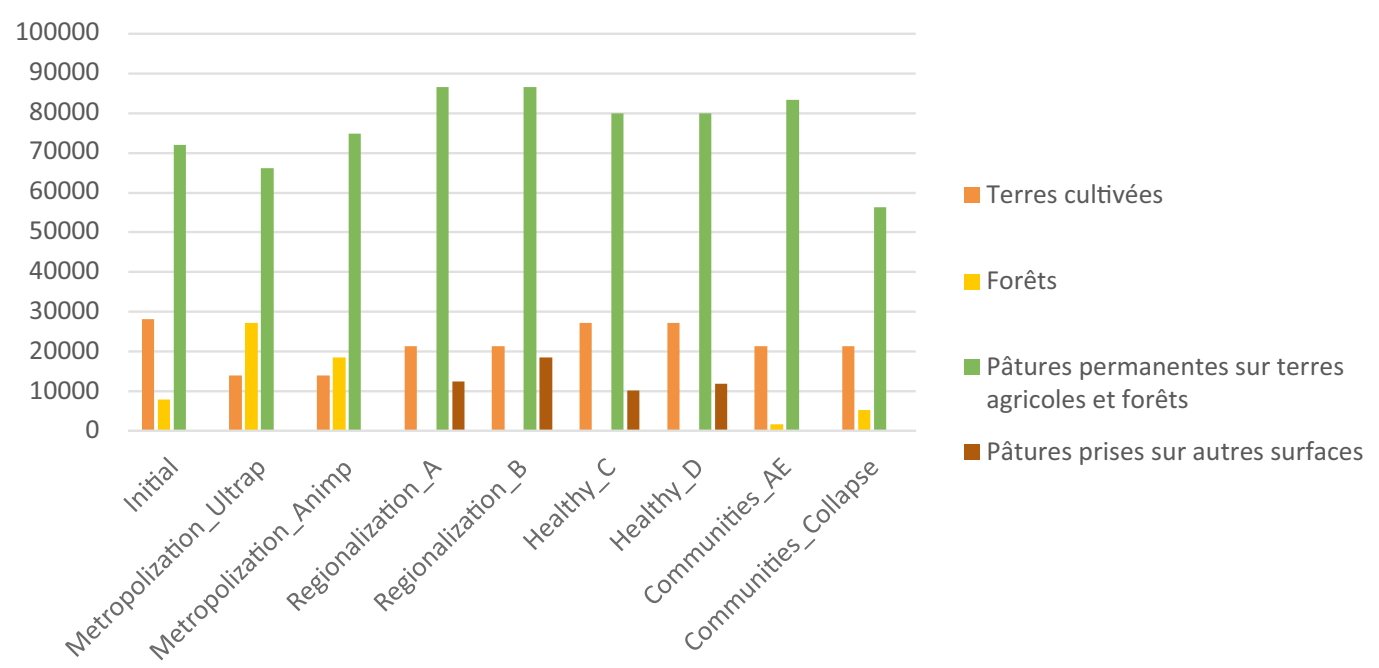

Fig. 4. Les superficies des terres cultivées, des forêts, des pâtures permanentes et des pâtures prises sur d'autres surfaces en Afrique du Nord en 2008 (initial) et avec quatre scénarios Agrimonde-Terra. Source : GlobaAgri-Ag-T.

moyenne dans toutes les régions du monde alors que dans la variante Healthy_D (agro-écologie), l'écart aux rendements potentiels diminue de $30 \%$ seulement.

Le scénario «Des usages des terres pour une alimentation de qualité et une nutrition saine » avec des hypothèses « agroécologie» pour les systèmes de cultures et d'élevage (Healthy_D) est très proche du scénario tunisien «Des usages des terres pour une alimentation diversifiée et de qualité et un système alimentaire territorialisé ». Le scénario «Des usages des terres pilotés par la métropolisation» est proche du scénario tunisien «Dualisme dans les usages des terres avec cohabitation de deux régimes alimentaires contrastés, de structures et de modes de production $\gg$.

En Afrique du Nord, en 2050, quel que soit le scénario Agrimonde-Terra, pour assurer les disponibilités alimentaires d'une population en forte croissance, les terres cultivées (cultures annuelles et permanentes) ne peuvent quasiment pas augmenter en raison de la faible disponibilité des terres arables. Les contraintes sur les terres sont très fortes.

Avec les deux variantes du scénario «Des usages des terres pilotés par la métropolisation », la dépendance commerciale est très forte, près du double de ce qu'elle est dans la situation initiale (Fig. 3). Dans ce scénario, il y a une transition alimentaire vers des produits ultra-transformés et des produits animaux, les marchés internationaux de produits agricoles sont très actifs en raison de la spécialisation agricole, et le changement climatique est accéléré. Les surfaces en forêts augmentent (Fig. 4) car des quantités importantes d'alimentation pour les hommes et les animaux sont importées.

Dans le scénario «Des usages des terres pour des systèmes alimentaires régionaux », l'arrêt des exportations de produits agricoles et le régime alimentaire «régional» limitent l'expansion des terres cultivées. L'Afrique du Nord ne pouvant cependant produire tout ce qu'elle consomme est contrainte d'importer (Fig. 3). Les surfaces en pâtures augmentent fortement sur les terres cultivées, les forêts, mais également sur les «autres surfaces » telles que les montagnes, le désert et les zones urbaines (Fig. 4).
Dans le scénario «Des usages des terres en tant que communs des communautés locales dans un monde fragmenté » (communities), la faible croissance économique rend le système très fragile et peu productif et conduit également à une croissance des importations (Fig. 3).

C'est avec le scénario «Des usages des terres pour une alimentation de qualité et une nutrition saine» avec les hypothèses d'intensification durable pour les cultures (Healthy_C) que la dépendance commerciale augmente le moins (Fig. 3). Elle augmente un peu plus la variante Healthy_D. En effet, l'évolution vers des régimes alimentaires sains, basés sur des céréales locales et des fruits et légumes et une faible augmentation de la consommation de produits animaux ainsi que la diversification des productions locales permettent de limiter les importations. La différence entre les deux variantes s'explique par les hypothèses sur les écarts de rendements potentiels et l'intensité culturelle. Par contre, dans ce scénario, les pâtures augmentent sur les terres cultivées et les superficies forestières et également sur les «autres surfaces». La gestion des pâtures est peu intensive ce qui permet de stocker du carbone dans les sols ; le sylvopastoralisme est pratiqué quand les conditions le permettent (Fig. 4).

Même si les conséquences des scénarios AgrimondeTerra sur les superficies et le commerce ont été simulées pour 1'Afrique du Nord et non pour la Tunisie, les tendances vont rester les mêmes pour la Tunisie. La pression sur les pâtures permanentes sera peut-être un peu plus faible que pour la moyenne de la région. On peut donc noter que c'est avec le scénario «Des usages des terres pour une alimentation de qualité et une nutrition saine» qui est proche du scénario tunisien «Des usages des terres pour une alimentation diversifiée et de qualité et un système alimentaire territorialisé » que la dépendance commerciale est la plus faible. Elle est plus élevée avec les hypothèses «agro-écologie» pour les systèmes de cultures et d'élevage (Healthy_D) qu'avec les hypothèses «Healthy_C». Ce résultat corrobore le choix des participants à l'atelier en Tunisie qui ont estimé que c'est avec le scénario «Des usages agro-écologiques des terres pour une alimentation diversifiée et de qualité et un système 
alimentaire territorialisé » que la dépendance commerciale aux importations serait la plus faible et que les terres cultivées se maintiendraient et contribueraient à la création d'emplois (Tab. 3).

\subsection{Comment la Tunisie peut-elle aller vers le scénario «Des usages agro-écologiques des terres pour une alimentation diversifiée et de qualité et un système alimentaire territorialisé »?}

Ce scénario sous-entend un changement de paradigme, c'est-à-dire produire mieux et durablement en préservant les ressources naturelles que sont l'eau et le sol, mais aussi en comprimant les coûts de production qui sont de plus en plus élevés et en assurant des emplois. Les projets de développement et de recherche-développement concernant le semis direct et l'agriculture de conservation ont commencé en Tunisie en 1999 grâce à des projets financés par le Fond français de l'environnement mondial (FFEM) (Raunet et al., 2004). Une étude comparative sur dix ans a démontré les bénéfices de cette technique (Angar et al., 2011). En 2016, une autre étude commanditée dans le cadre du projet «Agriculture de conservation au Maghreb », par l'association de Formation pour l'épanouissement et le renouveau de la terre (FERT) à l'APAD et l'ATAE, en collaboration étroite avec l'Institut national des grandes cultures (APAD et al., 2016) montre que les motifs conduisant à l'adoption de l'agriculture de conservation et les stratégies des agriculteurs sont de diverses natures. En effet, si certaines tendances sont favorables au développement de ce scénario, d'autres l'empêchent soit du fait d'intérêts divergents, soit par manque de volonté politique afin de mettre en place les préalables pour une telle trajectoire d'évolution.

\subsubsection{Les tendances favorables au développement de ce scénario}

Il y a tout d'abord une prise de conscience progressive de la nécessité de changer de modes de production de la part des agriculteurs dont les itinéraires techniques ne leur permettent plus de faire face aux effets du changement climatique et à la dégradation de leurs ressources. Cette prise de conscience a d'abord concerné les agriculteurs mécanisés disposant d'importantes assises foncières et ayant de ce fait une plus grande capacité de prise de risque. Parfois, l'engagement dans une trajectoire de transition est progressif, mais il est souvent le résultat d'un choc externe, comme une fluctuation des prix, une forte difficulté d'approvisionnement en intrants ou un événement climatique. Assez vite, certains d'entre eux ont pu rayonner sur leur voisinage et notamment sur des agriculteurs plus modestes en les amenant à adopter des techniques autant que des systèmes de production plus durables. L'accompagnement joue un rôle essentiel dans le cycle du changement (Bidaud, 2013). Les changements sont généralement progressifs, avec des retours en arrière partiels, et les situations ne sont jamais établies et souvent diverses, d'une exploitation à l'autre. Les agriculteurs se familiarisent progressivement avec les plantes, les sols, les animaux, les ravageurs, etc. de leurs exploitations. Ils deviennent des décideurs au lieu d'appliquer des produits et des techniques. Les résultats de l'enquête sur l'adoption du semis direct ont établi une typologie des agriculteurs. Quatre types de stratégies ont été révélées: les pionniers qui persévèrent et ont acquis une expérience et un savoir-faire significatifs; les essayants, nouveaux pratiquants de techniques telles que le semis direct, qui sont encore techniquement et/ou financièrement appuyés par des projets afin de maîtriser ces systèmes complexes. Les adaptants, ont quant à eux adopté une stratégie résultant des contraintes auxquelles ils ont dû faire face suite à l'introduction du semis direct. Enfin, il y a également des « renonçants » qui abandonnent les nouvelles pratiques et retournent à une agriculture conventionnelle (APAD et al., 2016).

Le caractère familial de l'agriculture tunisienne constitue un atout pour la diffusion d'une agriculture plus intégrée. Les tâches peuvent être distribuées entre plusieurs membres de la famille qui peuvent avoir des rôles complémentaires. Cette complémentarité peut améliorer les conditions de travail et de vie.

Le coût croissant des intrants rend aussi nécessaire leur substitution par des techniques qui utilisent les fonctionnalités écologiques et biologiques utiles présentes dans la nature pour les mettre au service de la production agricole.

Les villages et les territoires demeurent des lieux d'échanges dans lesquels une transformation des produits peut être réalisée et des circuits de proximité établis.

De même que, du côté des chercheurs, certains prennent conscience que les conditions de stress biotiques et abiotiques auxquelles sont soumises les cultures devraient être de plus en plus prises en compte dans les programmes de recherche et que de nouvelles formes de production plus résilientes sont à explorer. Ainsi, à titre d'exemple, les projets de recherche sur l'agriculture de conservation sont une réponse à la dégradation des ressources productives (eau, sol et couvert végétal). Les chercheurs impliqués dans ces projets estiment que pour faire face à cette dégradation une sortie du modèle classique est nécessaire, voire urgente, et que l'agriculture de conservation est sinon «la» solution, du moins une des solutions. De même du côté des consommateurs, et notamment de certaines associations de la société civile engagées dans les questions de nutrition et de santé humaine (telle que l'association de défense des consommateurs ODC), on assiste à une certaine sensibilité aux questions de qualité des produits et de leur impact sur la santé humaine. L'empreinte environnementale des produits alimentaires, même si elle n'est pas trop présente dans les débats publics, commence à émerger dans certains cercles de consommateurs avertis.

Les décideurs prennent aussi peu à peu conscience que les conditions du marché international ne sont plus favorables à une sécurité alimentaire soumise aux forces du marché et que la réponse pour préserver un niveau minimal de souveraineté alimentaire passe par un changement du modèle de production et une plus grande articulation entre les besoins alimentaires de la population et l'orientation productive de l'agriculture tunisienne. Cela se traduit par la mise en place de politiques de développement territorial basées sur la promotion des produits de terroir et de la multifonctionnalité de l'agriculture.

Ces facteurs sont favorables à un changement de paradigme, mais ne sont pas suffisamment forts pour que le changement ait réellement lieu. 
3.6.2 Les tendances qui empêchent le développement de cette trajectoire et les principaux leviers pour y faire face

Dans les faits et malgré la multiplication des expériences de modèles alternatifs d'agriculture davantage soucieux de durabilité (agriculture de conservation, agriculture biologique, agroforesterie, techniques douces de conservation des eaux et du sol, etc.) qui sont portées par plusieurs associations formelles ou informelles, l'agriculture tunisienne reste dominée par des formes d'intensification traditionnelle qui ont un impact négatif sur l'environnement et les ressources.

Plusieurs freins s'opposent au développement d'une telle alternative. Il y a tout d'abord le poids des tendances ellesmêmes. En effet, il est généralement admis que les changements de trajectoires sont difficiles. La mise en œuvre prend du temps, demande des connaissances sur les sols, sur les associations de cultures, ainsi que de nouveaux appuis institutionnels. Les résultats des recherches commencent à démontrer les divers avantages des pratiques agro-écologiques mais cette diversité surprend et ne convainc pas toujours. Sans accompagnement et formation, les agriculteurs ont tendance à conserver les pratiques auxquelles ils sont habitués. Les modifications de trajectoires demandent, par exemple, une bonne maîtrise technique pour le choix des dates de semis, des plantes de couverture, etc. et une bonne gestion de l'enherbement (Dugué, 2014). L'abandon du labour, souvent considéré comme le travail identitaire de l'agriculture, peut être également complexe; un agriculteur qui ne laboure plus serait considéré par ses pairs comme un mauvais agriculteur (Dugué, 2014).

Mais c'est aussi l'absence d'incitation sous ses différentes formes (production de biens publics, résultats de recherche par exemple, incitations financières, etc.) qui annihile la volonté de changer de trajectoire et l'adoption d'une nouvelle forme de production. Ainsi, par exemple, l'enquête sur l'agriculture de conservation en Tunisie (APAD et al., 2016) a montré que la pratique du semis direct semble stagner, voire régresser du fait de l'abandon de cette technique par de nombreux agriculteurs n'ayant pas pu gérer les effets indésirables de cette technique faute d'encadrement et de conseils adéquats. Ainsi en se basant sur les résultats d'une étude faite par l'INGC en 2010, les superficies conduites en semis direct qui couvraient 10000 à 12000 ha répartis entre une centaine d'agriculteurs semblent avoir régresser depuis suite à de multiples abandons (INGC, 2010, cité par l'étude APAD et al., 2016). Les agriculteurs en question signalent aussi l'absence d'une recherche appliquée qui propose des solutions adaptées au contexte tunisien avec ses conditions édapho-climatiques et sociales (la contrainte de la vaine pâture, par exemple).

Du côté des pouvoirs publics l'absence d'une politique de soutien d'un modèle alternatif en termes d'orientation des subventions aux producteurs, de soutien à la recherche ou encore aux organisations des producteurs ayant des pratiques qui préservent l'environnement constitue aussi un frein au développement d'une telle forme de production. L'État, par contre, soutient les céréales et les huiles de graines grâce à la Caisse générale de compensation (CGC). Celle-ci utilise l'Office des céréales pour maintenir à un niveau supérieur au marché international le prix payé au producteur et à un niveau inférieur le prix payé par l'ensemble des consommateurs, pour les produits de base (Rastoin et Benabderrazik, 2014). Pour les huiles de table, l'Office national des huiles (ONH) subventionne les prix à la consommation mais la production de graines oléagineuses n'est pas subventionnée (Rastoin et Benabderrazik, 2014).

Enfin, le fait que les entreprises de transformation et les consommateurs soient peu enclins à payer plus cher que les prix des produits conventionnels certains produits tels que les produits biologiques ou les produits de terroirs issus d'une agriculture plus raisonnée, limite la reconversion vers des trajectoires vertueuses qui peuvent concilier productivité et préservation de l'environnement, mais qui demandent du temps pour être mises en place.

Les Tunisiens consacrent encore une part importante de leur budget à l'alimentation: $35 \%$ en moyenne avec des différences notables entre les classes aisées (24\%) et pauvres $(52 \%)$ en 2005 (Rastoin et Benabderrazik, 2014). Les produits à base de céréales représentent encore une part importante des dépenses alimentaires. Le blé dur demeure la base de l'alimentation (semoule et pâtes) mais on observe une progression rapide du blé tendre (pain, biscuiterie, pâtisserie) avec l'occidentalisation du modèle de consommation (Rastoin et Benabderrazik, 2014). La dégradation de la qualité nutritionnelle de l'alimentaire avec une substitution de l'huile d'olive par l'huile de soja, de palme et de tournesol ainsi que l'augmentation de la consommation de produits gras et sucrés s'opposent au développement de l'approche agro-écologique. La Tunisie est confrontée à l'augmentation des maladies liées à la surnutrition et la persistance de carences nutritionnelles en raison de l'augmentation des revenus, des subventions accordées à certaines importations, de l'urbanisation croissante, de l'implantation d'un grand nombre de supermarchés, et également pour des raisons culturelles, car l'embonpoint a tendance à être socialement valorisé.

Enfin, l'écart important entre le niveau de la consommation et celui de la production nationale conduit la Tunisie à importer de grosses quantités de céréales: en moyenne 1,8 million de tonnes sur 2002-2012, avec d'importantes fluctuations en fonction de la pluviométrie locale. Sur ce total, le blé tendre représente environ $51 \%$, l'orge $25 \%$ et le blé dur $24 \%$.

Pour faire face à ces freins il est possible d'activer un certain nombre de leviers. Il y a tout d'abord la volonté politique qui doit saisir et donner corps à la prise de conscience des différents acteurs pour la mise en place d'un modèle alternatif qui reprend les fondements du scénario «Usages agro-écologiques des terres». Seulement un ensemble de politiques cohérentes et intégrées parviendront à modifier la trajectoire actuelle. Il faudrait à la fois des politiques alimentaires, rurales, environnementales et commerciales. Des alliances sont également possibles entre certains producteurs et certaines catégories de consommateurs afin de promouvoir ce nouveau modèle qui répond à la fois aux attentes des uns et des autres. Mais cette alliance n'a des chances de réussir que si elle mobilise les chercheurs afin qu'ils donnent un contenu technique, économique et social à ce modèle et que soient proposés des modes de gouvernance et un environnement institutionnel adéquats. Les chercheurs doivent développer des méthodologies de conception de systèmes agricoles innovants en intégrant les échelles de la parcelle, de l'exploitation agricole, du territoire et des filières et raisonner les changements d'échelle (Dugué, 2014). 


\section{Vers un chemin d'impact vers des usages durables des terres, une nutrition saine et une alimentation de qualité}

La construction de scénarios d'usages des terres en Tunisie a permis à l'équipe Agrimonde-Terra qui est intervenue de mieux réfléchir au chemin d'impact de la prospective pour que celle-ci permette de parvenir à un usage durable des terres qui bénéficie aux producteurs en leur assurant des revenus et des créations d'emplois, aux consommateurs en leur permettant d'avoir une alimentation plus saine et de qualité, qui permette l'amélioration de la qualité des sols, la fourniture de services écosystémiques, la production d'énergies renouvelables.

Un «output» d'Agrimonde-Terra est la tenue d'ateliers à l'échelle nationale et la construction de scénarios d'usages des terres et sécurité alimentaire à cette échelle.

Les «outcomes» de l'atelier et de la construction de scénarios à cette échelle sont:

- une analyse partagée par les participants de la situation actuelle des usages des terres et de la sécurité alimentaire et de ses enjeux; au début de l'atelier, les points de vue des participants étaient assez différents les uns des autres;

- une prise de conscience par les participants des déterminants du système «usages des terres et sécurité alimentaire », de leurs influences mutuelles et de leurs relations spatiales et temporelles;

- une intégration d'une partie de l'analyse faite pendant l'atelier par d'autres groupes ou études tels que le groupe de réflexion sur l'avenir de l'agriculture entre 2015 et 2035 et la stratégie de recherche qui doit l'accompagner, le programme de mise à niveau des exploitations agricoles en Tunisie, la stratégie du Synagri, les réflexions en cours sur les éventuelles implications de l'ALECA (accord de libre-échange complet et approfondi) entre la Tunisie et l'Union Européenne qui est en cours de négociations;

- un plaidoyer pour le changement.

Pour s'approprier le travail d'Agrimonde-Terra et construire des scénarios «Usages des terres et sécurité alimentaire » adaptés à la situation nationale, le groupe doit être divers (différentes disciplines, origines professionnelles, secteurs) et créatif (Cook et al., 2014). C'était le cas en Tunisie. Cependant, pour aboutir à des changements politiques, institutionnels, stratégiques ou techniques, des décideurs politiques et des acteurs économiques du système, y compris des agences de financement, doivent être impliqués dans l'identification des déterminants du système et la construction des scénarios (Wilkinson et Eidinow, 2008; Palazzo et al., 2016). La plupart des participants à l'atelier étaient des praticiens et non des décideurs politiques ou économiques, ce qui limite ou ralentit les changements. Il aurait été utile immédiatement après l'atelier des praticiens d'avoir un second atelier, plus court, avec des décideurs politiques afin de leur présenter les scénarios et leur comparaison et de progresser vers l'appui à la décision.

Par ailleurs, les participants à l'atelier se sont demandés si l'échelle nationale était la bonne et si de telles réflexions prospectives ne devraient pas être poursuivies avec des acteurs locaux pour mieux approfondir les moyens d'améliorer la sécurité foncière et les voies d'évolution des structures agricoles. L'utilité d'ateliers à l'échelle de grands territoires est à examiner.

Les impacts de niveau 1 pourront être de nouvelles réglementations foncières qui sécurisent l'accès à la terre, en particulier pour les jeunes et les femmes, des politiques alimentaires, des changements dans les accords commerciaux, des modifications dans les subventions accordées aux producteurs et aux consommateurs, de nouveaux programmes de recherche, l'acquisition de nouvelles compétences et des changements dans les pratiques des agriculteurs et des éleveurs grâce à des formations et du conseil, des alliances entre producteurs et consommateurs, des chaînes de valeur alimentaires localisées dans les territoires tunisiens, et l'adoption de systèmes de production durables, avec par exemple une amélioration de la fertilité des pâturages, un usage plus efficient des intrants, en particulier de l'eau.

Les impacts de niveau 2 pourront être des changements dans les cinq dimensions des usages des terres (par exemple, une diversification des productions, un accès plus sûr à la terre, une stabilisation du potentiel agronomique, des créations d'emplois dans l'agriculture et le secteur agro-alimentaire), ainsi qu'une augmentation des revenus des agriculteurs par la diversification des productions et des activités. Les impacts de niveau 2 pourront également se remarquer dans la sécurité alimentaire et nutritionnelle des populations, par exemple, une diminution du nombre de personnes en surpoids et des régimes alimentaires plus équilibrés.

Le fait que la démarche d'Agrimonde-Terra ait été rapidement appropriée par les participants tunisiens conduit à penser qu'il est possible à un groupe de décideurs nationaux d'utiliser les résultats de la prospective Agrimonde-Terra pour faire un diagnostic des usages des terres dans leur pays, construire des scénarios d'usages des terres et analyser leurs conséquences sur la sécurité alimentaire, définir une vision commune, faire une analyse stratégique des leviers nécessaires pour modifier les trajectoires en cours et aller vers des usages des terres et une sécurité alimentaire durable. La volonté des participants à l'atelier d'aller plus loin dans la réflexion prospective et dans l'action pour le changement témoigne de l'engagement de l'ensemble de la société tunisienne face aux enjeux actuels et futurs de l'agriculture et de la sécurité alimentaire du pays. L'agro-écologie nécessite un nouveau contrat de société avec une refonte des relations entre villes et campagnes ainsi qu'entre producteurs et consommateurs, un changement de la place de l'agriculture dans le modèle de développement, et un nouveau modèle alimentaire.

Remerciements. Les auteurs remercient tous les participants à l'atelier de construction de scénarios d'usages des terres et de sécurité alimentaire en Tunisie, ainsi que Véronique Lamblin (Futuribles) et Chloé Cangiano (étudiante, Agrosup Dijon) qui ont activement participé à la préparation et à l'animation de l'atelier. Ils remercient également tous les autres membres de l'équipe projet Agrimonde-Terra dont le travail a servi de base à la réflexion en Tunisie : Chantal Le Mouël (Inra, coordinatrice), Olivier Mora (Inra, organisateur de la construction des scénarios), Thierry Brunelle (Cirad), Catherine Donnars (Inra), Patrice Dumas (Cirad), Agneta Forslund (Inra), Stéphane Manceron (Inra), Elodie MarajoPetitzon (Inra) et Olivier Rechauchère (Inra). 


\section{Références}

Allan JA. 2003. Virtual water - the water, food, and trade nexus useful concept or misleading metaphor? Water Int 28: 1.

Amichi H, Jamin JY, Morardet S, et al. 2016. Le rôle du faire-valoir indirect dans le renouvellement générationnel des agriculteurs irrigants en Tunisie. Cah Agric 25 (3): 35004.

Angar H, Ben Haj Salah H, Ben-Hammouda M. Semis direct et semis conventionnel en Tunisie : les résultats agronomiques de 10 ans de comparaison. In : Bouzerzour $\mathrm{H}$, Irekti $\mathrm{H}$, Vadon $\mathrm{B}$, ed. 4èmes rencontres méditerranéennes du semis direct. Zaragoza (Espagne) : CIHEAM / ATU-PAM / INRAA / ITGC / FERT, 2011, vol. 96, pp. 53-59. Options Méditerranéennes : Série A. Séminaires Méditerranéen.

APAD, ATAE, FERT, INGC. 2016. Le semis direct en Tunisie : situation actuelle et perspectives. Étude réalisée dans le cadre du projet d'appui au développement de l'agriculture de conservation au Maghreb (Fert).

Badran M, Laher I. 2011. Obesity in arabic-speaking countries. $J$ Obes 2011: 1-9.

Ben Becher L. 2016. Tunisie : une nouvelle politique agricole pour relever les défis de la durabilité. CIHEAM Watch Lett 37.

Ben Saad A. 2014. Tunisie : inégalités de genre pour l'accès à la terre dans la région de Gafsa. CIHEAM Watch Lett 28. Land issues in the Mediterranean Countries.

Berger G. 1958. L'attitude prospective. Prospective 1: 4.

Bidaud F. 2013. Transitions vers la double performance: quelques approches sociologiques de la diffusion des pratiques agroécologiques. Centre d'études et de prospective. Analyse $n^{\circ} 63$, septembre 2013.

Chabbi M. 2006. L'urbanisation en Tunisie, transformations et tendances d'évolution. In : Villes réelles, villes projetées: Fabrication de la ville au Maghreb. Paris: Maisonneuve \& Larose.

Cirad, Inra. 2016. Agrimonde-Terra: foresight land use and food security in 2050. Short report of the foresight. Paris: Cirad and Inra. Available on : http://www.cirad.fr/publications-ressources/ edition/etudes-et-documents/prospective-agrimonde-terra et http:// institut.inra.fr/Missions/Eclairer-les-decisions/Prospectives/Tou tes-les-actualites/Prospective-Agrimonde-Terra (Accessed on 30 January 2017).

Cook CN, Inayatullah S, Burgman MA, Sutherland WJ, Wintle BA. 2014. Strategic foresight: how planning for the unpredictable can improve environmental decision-making. Trends Ecol Evol 29: 9. Available on: http://www.sciencedirect.com/science/article/pii/ S0169534714001566.

de Jouvenel B. 1964. L'Art de la conjecture. Monaco (Paris): Éditions du Rocher, SÉDÉIS (Société d'études et de documentation économiques, industrielles et sociales), coll. Futuribles.

de Jouvenel H. 2004. Invitation à la prospective. An invitation to foresight. Paris: Futuribles Perspectives.

de Lattre-Gasquet M, Moreau C. 2016. Un test de l'outil de dialogue Agrimonde-Terra : la construction de scénarios d'usages des terres en 2050 avec des décideurs tunisiens. Document de travail. Prospective Agrimonde-Terra : usage des terres et sécurité alimentaire en 2050. Disponible sur : http://www.cirad.fr/ publications-ressources/edition/etudes-et-documents/prospectiveagrimonde-terra et http://institut.inra.fr/Missions/Eclairer-les-deci sions/Prospectives/Toutes-les-actualites/Prospective-AgrimondeTerra (accès le 30 janvier 2017).

de Lattre-Gasquet M, Le Mouël C, Mora O. 2016. Agrimonde-Terra, a foresight exercise on land use and food security in 2050: scenario- building method and conceptual framework. Agrimonde-Terra, Brief No. 1.

Doligez F. 2015. Étude de reformulation concertée du programme de mise à niveau des exploitations agricoles en Tunisie. Argumentaire de politique publique présentant différents scénarios de mise à niveau des exploitations agricoles. Étude du ministère de l'Agriculture de Tunisie, de l'AFD et de l'IRAM. Rapport L4. Disponible sur: http://www.onagri.nat.tn/uploads/Etudes/RapportL4-MANAGRI-Tunisie_L4-Annexes_VDef_05-10-2015. pdf.

Dugué P. 2014. Semis direct, système de culture sur couverture végétale $(\mathrm{SCV})$ et agroécologie, vers où va-t-on? Altern Rural. Disponible sur: http://agritrop.cirad.fr/573006/1/docu ment_573006.pdf.

Elloumi M. Agriculture péri urbaine et nouvelles fonctions du foncier rural en Tunisie. In : Elloumi M, Jouve AM, Napoléone C, Paoli $\mathrm{JC}$, eds. Régulation foncière et protection des terres agricoles en méditerranée. Montpellier : CIHEAM, 2011, pp. 159-169. Options Méditerranéennes, Série B. Études et Recherches, $n^{\circ} 66$.

Elloumi M. 2013. Les terres domaniales en Tunisie. Études rurales 192: 43-60.

Elloumi M. Capacité de résilience de l'agriculture familiale tunisienne et politique agricole post révolution. In: Vianey G, Requier-Desjardins M, Paoli JC, eds. Accaparement, action publique, stratégies individuelles et ressources naturelles : regards croisés sur la course aux terres et à l'eau en contextes méditerranéens. Montpellier : CIHEAM, 2015, pp. 351-366. Options Méditerranéennes : Série B. Études et Recherches, $\mathrm{n}^{\circ} 72$.

Forward Thinking Platform (FTP). 2014. A glossary of terms commonly used in futures studies. Rome: Global Forum on Agricultural Research.

Gafrej R. 2016. Avec le changement climatique, quel avenir de l'agriculture en Tunisie ? CIHEAM Watch Lett 37.

Gasmi M. 2003. Accès des femmes rurales à la terre en Tunisie : entre le droit et le vécu. Atelier international «Femmes rurales et foncier ». Centre Forestier de Recyclage -Thiès, 25-27 février 2003, Sénégal.

Giannakopoulos C, Le Sager P, Bindi M, Moriondo M, Kostopoulou E, Goodess CM. 2009. Climatic changes and associated impacts in the mediterranean resulting from a $2^{\circ} \mathrm{C}$ global warming. Glob Planet Chang 68 (3): 209-224.

Godet M. 1997. Manuel de prospective stratégique. Paris : Dunod. Halle B, Allali A, Staatsen P. 2012. Profil environnemental de la Tunisie. Étude élaborée avec l'aide de l'Union européenne.

Hassen H, Croitoru L. 2015. Évaluation économique des biens et services des forêts tunisiennes. For Méditerr XXXIV: 4.

Jouve AM, Napoléone C. Modes de régulation de l'usage des terres en Méditerranée et protection des terres agricoles. In : Elloumi M, Jouve AM, Napoléone C, Paoli JC, eds. Régulation foncière et protection des terres agricoles en Méditerranée. Montpellier : CIHEAM, 2011. pp. 9-21 (Options Méditerranéennes : Série B. Études et Recherches, $\mathrm{n}^{\circ}$ 66). Séminaire FONCIMED sur les modes de régulation des échanges fonciers et de l'occupation du sol, 2008/10/08-12, Antalya (Turquie). Disponible sur : http://om. ciheam.org/om/pdf/b66/00801370.pdf.

Khamassi F, Bellagha S, Koussani W. 2016. Le système alimentaire tunisien : caractérisation et dynamiques. Les systèmes alimentaires territorialisés en Méditerranée. Initiatives pour une alimentation responsable et durable. $J$ Resolis 12.

Lahmar R. 2006. Opportunités et limites de l'agriculture de conservation en Méditerranée. Les enseignements du projet KASSA. In : Options Méditerranéennes. Zaragoza (Espagne): CIHEAMIAMZ. Série A, Vol. 69, pp. 11-18. 
Le Mouël C. 2016. Scenarios' simulation results. Technical Report. Agrimonde-Terra foresight: Land use and food security in 2050. Disponible sur : http://www.cirad.fr/publications-ressources/edi tion/etudes-et-documents/prospective-agrimonde-terra et http:// institut.inra.fr/Missions/Eclairer-les-decisions/Prospectives/Tou tes-les-actualites/Prospective-Agrimonde-Terra (Accessed on 30 January 2017).

Marty P. 2015. Le système alimentaire de la région Afrique du NordMoyen-Orient : une analyse retrospective 1961-2012. INRA, Rapport d'étude menée pour le compte de l'association Pluriagri.

Marx M, Fouquet B. 2013. Tunisie. Financement du secteur agricole. Centre d'investissement de la FAO, étude $\mathrm{n}^{\circ} 9$.

Ministère de l'Agriculture et de l'Environnement de Tunisie, AFD, CIRAD, GRET, IRAM. 2011. Actualisation concertée de la politique agricole. Orientations pour un nouvel agenda agricole Tunisien. Disponible sur : http://www.afd.fr/webdav/shared/ PORTAILS/PAYS/TUNISIE/Publication\%20Tunisie/TunisiePOLAGRI_2ieme edition.pdf.

Mokhtar A. 2013. L'adaptation aux changements climatiques basés sur les écosystèmes, l'exemple de la Tunisie. For Méditerr XXXIV: 4.

Moreau C, Cangiano C, de Lattre-Gasquet M. 2015. Usages des terres et sécurité alimentaire en 2050. Cas d'étude tunisien. Document de travail.

ONAGRI. 2016. Le foncier agricole en Tunisie : situation et contraintes de mise en valeur. La lettre de l'ONAGRI 2(4).

Palazzo A, et al. 2016. Linking regional stakeholder scenarios and shared socioeconomic pathways: quantified west African food and climate futures in a global context. Glob Environ Chang. Disponible sur: https://doi.org/10.1016/j.gloenvcha.2016.12.002.
Picouet M. 2006. Dynamique des populations, disponibilités en terres et adaptation des régimes fonciers. Le cas de la Tunisie. Paris : Comité international de coopération dans les recherches nationales en démographie (CICRED).

Rastoin JL, Benabderrazik EH. 2014. Céréales et oléoprotéagineux. Construire la Méditerranée. IPEMED.

Raunet M, Richard JF, Rojat D. Premiers résultats d'introduction du semis direct sous couvert et lutte antiérosive en Tunisie. In : Roose E, De Noni G, Prat C, Ganry F, Bourgeon G, IRD, CIRADAMIS-AGRONOMIE. Gestion de la biomasse, érosion et séquestration du carbone. Séquestration du carbone et érosion des sols. Paris : IRD, 2004, pp. 388-404 (Bulletin du réseau érosion, 23).

Roose E, ed. 2015. Restauration de la productivité des sols tropicaux et méditerranéens. Contribution à l'agroécologie. Version préliminaire. Montpellier : IRD éditions.

Van Witzke H, Noleppa S. 2010. EU agricultural production and trade: can more efficiency prevent increasing 'land grabbing' outside Europe? Università Cattolica del Sacro Cuore, Piacenza: OPERA.

Wilkinson A, Eidinow E. 2008. Evolving practices in environmental scenarios: a new scenario typology. Environ Res Lett 3: 45017.

Zaghouane O, Abdellaoui Z, Houassine D. Quelles perspectives pour l'agriculture de conservation dans les zones céréalières en conditions algériennes ? In : Options Méditerranéennes. Zaragoza (Espagne) : CIHEAM-IAMZ, 2006, série A, Vol. 69, pp. 183-187.

Zdruli P. 2014. Land resources of the Mediterranean: status, pressures, trends and impacts on future regional development. Land Degrad Develop 25: 373-384.

Citation de l'article : de Lattre-Gasquet M, Moreau C, Elloumi M, Ben Becher L. 2017. Vers un scénario « Des usages agro-écologiques des terres pour une alimentation diversifiée et de qualité et un système alimentaire territorialisé » en Tunisie en 2050. OCL 24(3): D306. 